\title{
Amenability of ultrapowers of Banach algebras
}

\author{
Matthew Daws
}

November 20, 2018

\begin{abstract}
We study when certain properties of Banach algebras are stable under ultrapower constructions. In particular, we consider when every ultrapower of $\mathcal{A}$ is Arens regular, and give some evidence that this is so if and only if $\mathcal{A}$ is isomorphic to a closed subalgebra of operators on a super-reflexive Banach space. We show that such ideas are closely related to whether one can sensibly define an ultrapower of a dual Banach algebra. We study how tensor products of ultrapowers behave, and apply this to study the question of when every ultrapower of $\mathcal{A}$ is amenable. We provide an abstract characterisation in terms of something like an approximate diagonal, and consider when every ultrapower of a $\mathrm{C}^{*}$-algebra, or a group $L^{1}$-convolution algebra, is amenable.

2000 Mathematics Subject Classification: Primary 46B08, 46B28, 46H05, 43A20; Secondary 22D15, 47L10, 46M05, 46M07

Keywords: Banach algebra, Arens products, ultrapower, amenability, tensor product.
\end{abstract}

\section{Introduction}

Given a Banach space $E$ and an ultrafilter $\mathcal{U}$, we can form the ultrapower $(E)_{\mathcal{U}}$. This construction has proved to be useful in Banach space theory, especially with regards to local theory. Given a Banach algebra $\mathcal{A}$, it is trivial that $(\mathcal{A})_{\mathcal{U}}$ is a Banach algebra. Right at the beginning of the study of ultrapowers, in [6], certain sequence spaces which are Banach algebras were studied. As noted in [16], $\mathrm{C}^{*}$-algebra techniques can be used to show that the class of $C(K)$ spaces is closed under ultrapower constructions. In [15], [17] and [7, ultrapowers of Banach algebras were used to study the Arens products on the bidual of $\mathcal{A}$. In [14, Ge and Hadwin study ultrapowers of $\mathrm{C}^{*}$-algebras. Otherwise, the study of ultrapowers of Banach algebras has been surprisingly sparse (see below for further points).

For a property of Banach spaces $(P)$, we say that a Banach space has super- $(P)$ if every ultrapower of $E$ has $(P)$. The best known example is that of a super-reflexive Banach space (see [16. Section 6]). We shall study some super properties of Banach algebras: in particular, when ultrapowers of a Banach algebra are Arens regular, and when they are amenable.

There seems to be a close relationship between a Banach algebra being super Arens regular, and the algebra being isomorphic to a closed subalgebra of operators on a super-reflexive Banach space. We also show that the natural construction of an ultrapower of a dual Banach algebra only works, in practice, for super Arens regular Banach algebras.

We say that a Banach algebra $\mathcal{A}$ is ultra-amenable if every ultrapower of $\mathcal{A}$ is amenable (the term super-amenable is used for another meaning by Runde in [30]). We show that ultra-amenability is strictly weaker than contractability (which is what Runde calls super-amenable), and strictly stronger than amenability. Part of our motivation is that it is generally easy to show that a Banach algebra is not contractible, while amenability is a much harder property to settle (this applies in particular to $\mathcal{B}(E)$, the algebra of operators on a Banach space $E$ ). We hope that perhaps the ultra-amenability of $\mathcal{B}(E)$ can be more easily settled, although our current techniques do not allow this. 
We provide an abstract characterisation of ultra-amenability, similar to the concept of an approximate diagonal, see [30, Section 2.2]. To do this, we need to first study how tensor products and ultrapowers interact. We present a counter-example due to Charles Read that ultrapowers and tensor products do not "commute". We settle when a $\mathrm{C}^{*}$-algebra is ultra-amenable, and show for many locally compact groups $G$ that ultra-amenability is equivalent to being finite.

\subsection{Notation and basic concepts}

We generally follow 3 , for notation and Banach algebra concepts. Let $E$ be a Banach space. We write $E^{\prime}$ for the dual space of $E$, and for $x \in E$ and $\mu \in E^{\prime}$, we write $\langle\mu, x\rangle$ for $\mu(x)$. We occasionally use square brackets for inner-products. Recall the canonical map $\kappa_{E}: E \rightarrow E^{\prime \prime}$ defined by $\left\langle\kappa_{E}(x), \mu\right\rangle=\langle\mu, x\rangle$ for $x \in E$ and $\mu \in E^{\prime}$. When $\kappa_{E}$ is an isomorphism, we say that $E$ is reflexive.

Recall the notions of filter and ultrafilter. Let $\mathcal{U}$ be a non-principal ultrafilter on a set $I$, and let $E$ be a Banach space. We form the Banach space

$$
\ell^{\infty}(E, I)=\left\{\left(x_{i}\right)_{i \in I} \subseteq E:\left\|\left(x_{i}\right)\right\|:=\sup _{i \in I}\left\|x_{i}\right\|<\infty\right\},
$$

and define the closed subspace

$$
\mathcal{N}_{\mathcal{U}}=\left\{\left(x_{i}\right)_{i \in I} \in \ell^{\infty}(E, I): \lim _{i \rightarrow \mathcal{U}}\left\|x_{i}\right\|=0\right\} .
$$

Thus we can form the quotient space, called the ultrapower of $E$ with respect to $\mathcal{U}$,

$$
(E)_{\mathcal{U}}:=\ell^{\infty}(E, I) / \mathcal{N}_{\mathcal{U}}
$$

In general, this space will depend on $\mathcal{U}$, though many properties of $(E)_{\mathcal{U}}$ turn out to be independent of $\mathcal{U}$, as long as $\mathcal{U}$ is sufficiently "large" in some sense.

We can verify that, if $\left(x_{i}\right)_{i \in I}$ represents an equivalence class in $(E)_{\mathcal{U}}$, then

$$
\left\|\left(x_{i}\right)_{i \in I}+\mathcal{N}_{\mathcal{U}}\right\|=\lim _{i \rightarrow \mathcal{U}}\left\|x_{i}\right\| .
$$

We shall abuse notation and write $\left(x_{i}\right)$ for the equivalence class it represents; of course, it can be checked that any definition we make is independent of the choice of representative of the equivalence class. There is a canonical isometry $E \rightarrow(E)_{\mathcal{U}}$ given by sending $x \in E$ to the constant family $(x)$. We again abuse notation and write $x \in(E)_{\mathcal{U}}$, identifying $E$ with a closed subspace of $(E)_{\mathcal{U}}$.

Definition 1.1. An ultrafilter $\mathcal{U}$ is countably incomplete when there exists a sequence $\left(U_{n}\right)_{n=1}^{\infty}$ in $\mathcal{U}$ such that $U_{1} \supseteq U_{2} \supseteq U_{3} \supseteq \cdots$ and such that $\bigcap_{n} U_{n}=\emptyset$.

Countably incomplete ultrafilters are useful, because they allow us to embed sequential convergence into convergence along the ultrafilter (see numerous examples of this argument in [16]). We remark that if there exists a non-countably incomplete ultrafilter, then there exists an uncountable measurable cardinal, and it is known that the existence of such cardinals cannot be shown in ZFC. See [1, Section 4.2] for further details. Notice that any non-principal ultrafilter on a countable index set is certainly countably incomplete.

There is a canonical map $\left(E^{\prime}\right)_{\mathcal{U}} \rightarrow(E)_{\mathcal{U}}^{\prime}$ given by

$$
\left\langle\left(\mu_{i}\right),\left(x_{i}\right)\right\rangle=\lim _{i \rightarrow \mathcal{U}}\left\langle\mu_{i}, x_{i}\right\rangle \quad\left(\left(\mu_{i}\right) \in\left(E^{\prime}\right)_{\mathcal{U}},\left(x_{i}\right) \in(E)_{\mathcal{U}}\right) .
$$

This map is an isometry, and so we identify $\left(E^{\prime}\right)_{\mathcal{U}}$ with a closed subspace of $(E)_{\mathcal{U}}^{\prime}$. It is shown in [16, Proposition 7.1] that when $\mathcal{U}$ is countably incomplete, $(E)_{\mathcal{U}}^{\prime}=\left(E^{\prime}\right)_{\mathcal{U}}$ if and only if $(E)_{\mathcal{U}}$ is 
reflexive. Furthermore, we define a Banach space $E$ to be super-reflexive if $(E)_{\mathcal{U}}$ is reflexive for any ultrafilter $\mathcal{U}$. As shown in [16, Proposition 6.4], this definition is equivalent to the original one given by James (see [18]).

For Banach spaces $E$ and $F$, we write $\mathcal{B}(E, F)$ for the space of bounded linear operators from $E$ to $F$. Then there is a canonical isometric map $(\mathcal{B}(E, F))_{\mathcal{U}} \hookrightarrow \mathcal{B}\left((E)_{\mathcal{U}},(F)_{\mathcal{U}}\right)$ given by

$$
T(x)=\left(T_{i}\left(x_{i}\right)\right) \quad\left(T=\left(T_{i}\right) \in(\mathcal{B}(E, F))_{\mathcal{U}}, x=\left(x_{i}\right) \in(E)_{\mathcal{U}}\right) .
$$

We shall often identify $(\mathcal{B}(E, F))_{\mathcal{U}}$ with its image in $\mathcal{B}\left((E)_{\mathcal{U}},(F)_{\mathcal{U}}\right)$.

\section{Basics of ultrapowers of Banach algebras}

When $\mathcal{A}$ is a Banach algebra, $(\mathcal{A})_{\mathcal{U}}$ becomes a Banach algebra under the pointwise product. This follows, as it is easy to show that $\mathcal{N}_{\mathcal{U}}$ is a closed ideal in the Banach algebra $\ell^{\infty}(\mathcal{A}, I)$.

In [14, Ge and Hadwin make a general study of ultrapowers of $\mathrm{C}^{*}$-algebras. Much of what they prove can easily be adapted to general Banach algebras. To give just one example, the ideas of [14, Section 3] will show that if $\mathcal{A}$ is a separable Banach algebra and $\mathcal{U}$ and $\mathcal{V}$ are non-principal ultrafilters on $\mathbb{N}$, then $(\mathcal{A})_{\mathcal{U}}$ and $(\mathcal{A})_{\mathcal{V}}$ are isomorphic as Banach algebras, assuming the continuum hypothesis holds.

Proposition 2.1. For a Banach algebra $\mathcal{A}$, an ultrapower $(\mathcal{A})_{\mathcal{U}}$ is unital if and only if $\mathcal{A}$ is unital.

Proof. Clearly, if $\mathcal{A}$ is unital, then so is $(\mathcal{A})_{\mathcal{U}}$. Let $e=\left(e_{i}\right) \in(\mathcal{A})_{\mathcal{U}}$ be a unit for $(\mathcal{A})_{\mathcal{U}}$, and choose $e_{i}$ such that $\left\|e_{i}\right\|=\|e\| \geq 1$ for each $i$. For $\epsilon>0$, for each $i$, let $a_{i} \in \mathcal{A}$ be such that $\left\|a_{i}\right\|=1$ and

$$
\left\|a_{i}-e_{i} a_{i}\right\| \geq \sup \left\{\left\|a-e_{i} a\right\|: a \in \mathcal{A},\|a\| \leq 1\right\}-\epsilon
$$

Let $b=\left(a_{i}\right) \in(\mathcal{A})_{\mathcal{U}}$, so that $b=e b=\left(e_{i} a_{i}\right) \in(\mathcal{A})_{\mathcal{U}}$, and hence

$$
0=\lim _{i \rightarrow \mathcal{U}}\left\|a_{i}-e_{i} a_{i}\right\| \geq \lim _{i \rightarrow \mathcal{U}} \sup \left\{\left\|a-e_{i} a\right\|: a \in \mathcal{A},\|a\| \leq 1\right\}-\epsilon .
$$

As $\epsilon>0$ was arbitrary, we see that

$$
\lim _{i \rightarrow \mathcal{U}} \sup \left\{\left\|a-e_{i} a\right\|: a \in \mathcal{A},\|a\| \leq 1\right\}=0 .
$$

Analogously, we see that

$$
\lim _{i \rightarrow \mathcal{U}} \sup \left\{\left\|a-a e_{i}\right\|: a \in \mathcal{A},\|a\| \leq 1\right\}=0 .
$$

For $\epsilon>0$, let $U=\left\{i:\left\|a-a e_{i}\right\|+\left\|a-e_{i} a\right\|<\epsilon(a \in \mathcal{A},\|a\| \leq 1)\right\} \in \mathcal{U}$. Thus, for $i, j \in U$,

$$
\left\|e_{i}-e_{j}\right\| \leq\left\|e_{i}-e_{i} e_{j}\right\|+\left\|e_{j}-e_{i} e_{j}\right\|<2 \epsilon\|e\| .
$$

It is straightforward to extract a sequence from the family $\left(e_{i}\right)$ which will be Cauchy, and hence converges to, say, $e_{\mathcal{A}} \in \mathcal{A}$. It is then clear that $e_{\mathcal{A}}$ will be a unit for $\mathcal{A}$.

The following is perhaps a little more surprising.

Proposition 2.2. For a Banach algebra $\mathcal{A}$, an ultrapower $(\mathcal{A})_{\mathcal{U}}$ has a bounded approximate identity if and only if $\mathcal{A}$ does. The same statement holds for left or right bounded approximate identities. 
Proof. Suppose that $\mathcal{A}$ has a bounded approximate identity of bound $M \geq 1$. Let $\mathcal{U}$ be an ultrafilter on an index set $I$, let $a=\left(a_{i}\right) \in(\mathcal{A})_{\mathcal{U}}$, and let $\epsilon>0$. Again, we may suppose that $\left\|a_{i}\right\|=\|a\|$ for each $i \in I$. For each $i \in I$, we can find $u_{i} \in \mathcal{A}$ with $\left\|u_{i}\right\| \leq M$ and $\left\|a_{i}-u_{i} a_{i}\right\|<\epsilon$. Let $u=\left(u_{i}\right)_{i \in I} \in(\mathcal{A})_{\mathcal{U}}$, so that $\|a-u a\| \leq \epsilon$. As $a$ and $\epsilon$ were arbitrary, we see that $(\mathcal{A})_{\mathcal{U}}$ has bounded left approximate units. By [3, Corollary 2.9.15], we have that $(\mathcal{A})_{\mathcal{U}}$ has a bounded left approximate identity of bound $M$. By symmetry, $(\mathcal{A})_{\mathcal{U}}$ has a bounded right approximate identity of bounded $M$, and so by a result due to Dixon, see [3. Proposition 2.9.3], we have that $(\mathcal{A})_{\mathcal{U}}$ has a bounded approximate identity of bound $2 M+M^{2}$.

Conversely, suppose that $(\mathcal{A})_{\mathcal{U}}$ has a bounded approximate identity of bound $M$, but that $\mathcal{A}$ does not have a bounded left approximate identity of bound $\leq M$. Hence $\mathcal{A}$ does not have bounded left approximate units of bound $\leq M$. In particular, there exists $a \in \mathcal{A}$ and $\delta>0$ such that $\|a-u a\| \geq \delta$ for all $u \in \mathcal{A}$ with $\|u\| \leq M$. However, we can find $u=\left(u_{i}\right) \in(\mathcal{A})_{\mathcal{U}}$ with $\|a-u a\|<\delta / 2$, that is, $\lim _{i \rightarrow \mathcal{U}}\left\|a-u_{i} a\right\|<\delta / 2$, a contradiction. So $\mathcal{A}$ has a bounded left approximate identity of bound $M$, and thus by symmetry, $\mathcal{A}$ has a bounded approximate identity.

Ultrapowers have been studied in the context of von Neumann algebras. However, here the definition is different to ours: this is because, for example, if $\mathcal{M}=\ell^{\infty}$ and $\mathcal{U}$ is a non-principal ultrafilter on $\mathbb{N}$, then $(\mathcal{M})_{\mathcal{U}}$ is not a dual space, and hence not a von Neumann algebra. Instead, a construction using traces is often used; however, it can be shown that the predual of the von Neumann algebra ultrapower is precisely the Banach space ultrapower of the predual (see, for example, [28, Section 1]). We study such ideas for dual Banach algebras below.

Ultrapowers of Banach spaces have been used in [2] to study representations of Banach algebras and representations of groups; see also the similar ideas used in [10] and [29].

\section{Arens regularity}

Let $\mathcal{A}$ be a Banach algebra. We now recall the Arens products on $\mathcal{A}^{\prime \prime}$. Firstly, we turn $\mathcal{A}^{\prime}$ into a $\mathcal{A}$-bimodule in the usual fashion,

$$
\langle a \cdot \mu, b\rangle=\langle\mu, b a\rangle, \quad\langle\mu \cdot a, b\rangle=\langle\mu, a b\rangle \quad\left(a, b \in \mathcal{A}, \mu \in \mathcal{A}^{\prime}\right) .
$$

In a similar way, $\mathcal{A}^{\prime \prime}$ and so forth also become $\mathcal{A}$-bimodules. Then we define bilinear maps $\mathcal{A}^{\prime \prime} \times$ $\mathcal{A}^{\prime}, \mathcal{A}^{\prime} \times \mathcal{A}^{\prime \prime} \rightarrow \mathcal{A}^{\prime}$ by

$$
\langle\Phi \cdot \mu, a\rangle=\langle\Phi, \mu \cdot a\rangle, \quad\langle\mu \cdot \Phi, a\rangle=\langle\Phi, a \cdot \mu\rangle \quad\left(\Phi \in \mathcal{A}^{\prime \prime}, \mu \in \mathcal{A}^{\prime}, a \in \mathcal{A}\right) .
$$

Finally, we define bilinear maps $\square, \diamond: \mathcal{A}^{\prime \prime} \times \mathcal{A}^{\prime \prime} \rightarrow \mathcal{A}^{\prime \prime}$ by

$$
\langle\Phi \square \Psi, \mu\rangle=\langle\Phi, \Psi \cdot \mu\rangle, \quad\langle\Phi \diamond \Psi, \mu\rangle=\langle\Psi, \mu \cdot \Phi\rangle \quad\left(\Phi, \Psi \in \mathcal{A}^{\prime \prime}, \mu \in \mathcal{A}^{\prime}\right) .
$$

These are associative products which extend the natural action of $\mathcal{A}$ on $\mathcal{A}^{\prime \prime}$, called the first and second Arens products. See [3, Section 3.3] or [22, Section 1.4] for further details. Thus $\square$ and $\diamond$ agree with the usual product on $\kappa_{\mathcal{A}}(\mathcal{A})$. When $\square$ and $\diamond$ agree on all of $\mathcal{A}^{\prime \prime}$, we say that $\mathcal{A}$ is Arens regular.

By Goldstein's Theorem, we know that the unit ball of $\mathcal{A}$ is weak*-dense in the unit ball of $\mathcal{A}^{\prime \prime}$. This allows us to find an ultrafilter $\mathcal{U}$ such that, given $\Phi, \Psi \in \mathcal{A}^{\prime \prime}$, we can find bounded families $\left(a_{i}\right)$ and $\left(b_{i}\right)$ with $\left(a_{i}\right)$ tending to $\Phi$ weak* along $\mathcal{U}$, and $\left(b_{i}\right)$ tending to $\Psi$. See [16, Proposition 6.7] for further details. Then

$$
\langle\Phi \square \Psi, \mu\rangle=\lim _{j \rightarrow \mathcal{U}} \lim _{i \rightarrow \mathcal{U}}\left\langle\mu, a_{i} b_{j}\right\rangle, \quad\langle\Phi \diamond \Psi, \mu\rangle=\lim _{i \rightarrow \mathcal{U}} \lim _{j \rightarrow \mathcal{U}}\left\langle\mu, a_{i} b_{j}\right\rangle \quad\left(\mu \in \mathcal{A}^{\prime}\right) .
$$


In [7] we show that when $\mathcal{A}$ is Arens regular, we can find a more "symmetric" version of these formulae.

We shall say that $\mathcal{A}$ is super Arens regular if every ultrapower of $\mathcal{A}$ is Arens regular. As Arens regularity passes to subalgebras, clearly a super Arens regular Banach algebra is Arens regular.

Proposition 3.1. Let $\mathcal{A}$ be a Banach algebra isomorphic to a closed subalgebra of $\mathcal{B}(E)$ for a super-reflexive Banach space $E$. Then $\mathcal{A}$ is super Arens regular.

Proof. It is shown in [10] that $\mathcal{B}(E)$ is Arens regular for any super-reflexive Banach space $E$. Let $\mathcal{U}$ be an ultrafilter. As an ultrapower of an ultrapower is again an ultrapower (see [16, Page 90]), we see that $(E)_{\mathcal{U}}$ is super-reflexive. We identify $(\mathcal{B}(E))_{\mathcal{U}}$ as a closed subalgebra of $\mathcal{B}\left((E)_{\mathcal{U}}\right)$. Thus $(\mathcal{B}(E))_{\mathcal{U}}$ is Arens regular, and hence so is $(\mathcal{A})_{\mathcal{U}}$, as required.

Note that if $\mathcal{A}$ is a Banach algebra whose underlying Banach space is super-reflexive, then every ultrapower of $\mathcal{A}$ is reflexive, and hence certainly Arens regular. As noted in [10], if $\mathcal{A}$ is a closed subalgebra of $\mathcal{B}(E)$ for a super-reflexive $E$ (or $\mathcal{A}$ is super-reflexive) then every even dual of $\mathcal{A}$ is Arens regular.

Let $\mu \in \mathcal{A}^{\prime}$. We say that $\mu$ is weakly almost periodic if the map

$$
L_{\mu}: \mathcal{A} \rightarrow \mathcal{A}^{\prime} ; \quad a \mapsto a \cdot \mu \quad(a \in \mathcal{A})
$$

is weakly compact, and write $\mu \in \operatorname{WAP}\left(\mathcal{A}^{\prime}\right)$. Then $\mathcal{A}$ is Arens regular if and only if $\operatorname{WAP}\left(\mathcal{A}^{\prime}\right)=$ $\mathcal{A}^{\prime}$. See [4, Section 3] for further details (and be aware that they write $\operatorname{WAP}(\mathcal{A})$ ). A useful characterisation of $\operatorname{WAP}\left(\mathcal{A}^{\prime}\right)$, due originally to John Pym (see [27, Theorem 4.3]), is that $\mu \in$ $\operatorname{WAP}\left(\mathcal{A}^{\prime}\right)$ if and only if $\langle\Phi \square \Psi, \mu\rangle=\langle\Phi \diamond \Psi, \mu\rangle$ for all $\Phi, \Psi \in \mathcal{A}^{\prime \prime}$. Combining this fact with some careful arguments yields the following repeated limit criterion.

Proposition 3.2. Let $\mathcal{A}$ be a Banach algebra, and let $\mu \in \mathcal{A}^{\prime}$. Then $\mu$ is weakly almost periodic if and only if, for bounded sequences $\left(a_{n}\right)$ and $\left(b_{m}\right)$ in $\mathcal{A}$, we have that

$$
\lim _{n \rightarrow \infty} \lim _{m \rightarrow \infty}\left\langle\mu, a_{n} b_{m}\right\rangle=\lim _{m \rightarrow \infty} \lim _{n \rightarrow \infty}\left\langle\mu, a_{n} b_{m}\right\rangle,
$$

whenever all the iterated limits exist.

Proof. See [3, Theorem 2.6.17] or [4, Section 3], for example.

For an ultrapower $(\mathcal{A})_{\mathcal{U}}$, we generally do not fully understand the dual $(\mathcal{A})_{\mathcal{U}}^{\prime}$. However, we have the norming subspace $\left(\mathcal{A}^{\prime}\right)_{\mathcal{U}}$, and so in particular, if $(\mathcal{A})_{\mathcal{U}}$ is Arens regular, then $\left(\mathcal{A}^{\prime}\right)_{\mathcal{U}} \subseteq$ $\operatorname{WAP}\left((\mathcal{A})_{\mathcal{U}}^{\prime}\right)$.

Lemma 3.3. Let $\mathcal{A}$ be a Banach algebra, and let $\mathcal{U}$ be a countably incomplete ultrafilter. An ultrapower $(\mathcal{A})_{\mathcal{U}}$ is Arens regular if and only if $\left(\mathcal{A}^{\prime}\right)_{\mathcal{U}} \subseteq \operatorname{WAP}\left((\mathcal{A})_{\mathcal{U}}^{\prime}\right)$.

Proof. We need only show the "if" part. Let $\mu \in(\mathcal{A})_{\mathcal{U}}^{\prime}$, and suppose that $\mu$ is not weakly almost periodic. Thus there exist bounded sequences $\left(a_{n}\right)$ and $\left(b_{m}\right)$ in $(\mathcal{A})_{\mathcal{U}}$ such that the iterated limits of $\left(\left\langle\mu, a_{n} b_{m}\right\rangle\right)$ exist, but are not equal. Let $E \subseteq(\mathcal{A})_{\mathcal{U}}$ be the closed linear span of $\left(a_{n} b_{m}\right)_{n, m \in \mathbb{N}}$, so that $E$ is separable. As $\mathcal{U}$ is countably incomplete, we can apply [16, Corollary 7.5] to see that there exists $\lambda \in\left(\mathcal{A}^{\prime}\right)_{\mathcal{U}}$ such that $\left\langle\mu, a_{n} b_{m}\right\rangle=\left\langle\lambda, a_{n} b_{m}\right\rangle$ for all $n$ and $m$. Thus $\lambda$ is not weakly almost periodic, a contradiction.

For a Banach space $E$, let $\mathcal{F}(E)$ be the space of finite-rank operators on $E$, and let $\mathcal{A}(E)$ be the space of approximable operators, the norm closure of $\mathcal{F}(E)$ in $\mathcal{B}(E)$. See below for further details, and for what it means for a Banach space $E$ to have the approximation property. It is known that if $E$ is a reflexive Banach space with the approximation property, then $\mathcal{A}(E)$ is Arens regular, and that $\mathcal{A}(E)^{\prime \prime}=\mathcal{B}(E)$ as a Banach algebra (see, for example, [21] or [22, Section 1.7]). In general, $\mathcal{A}(E)$ is Arens regular if and only if $E$ is reflexive (see [3, Theorem 2.6.23], for example). 
Proposition 3.4. Let $E$ be a Banach space. The $\mathcal{A}(E)$ is super Arens regular if and only if $E$ is super-reflexive.

Proof. By the above, if $E$ is super-reflexive, then $\mathcal{A}(E)$ is super Arens regular. If $E$ is not superreflexive, then by the results of [16, Section 6], there exists a countably incomplete ultrafilter $\mathcal{U}$ on an index set $I$ such that $(E)_{\mathcal{U}}$ is not reflexive. By a result of James (see, for example, [10, Section 4]), we can find bounded sequences $\left(x^{(n)}\right)$ in $(E)_{\mathcal{U}}$ and $\left(\mu^{(m)}\right)$ in $(E)_{\mathcal{U}}^{\prime}$ such that

$$
\left\langle\mu^{(m)}, x^{(n)}\right\rangle= \begin{cases}0 & : m>n, \\ 1 & : m \leq n .\end{cases}
$$

Let $E$ be the closed linear span of the $\left(x^{(n)}\right)$, so that $E$ is separable. As we only care about the value of $\mu^{(m)}$ on $E$, by [16, Corollary 7.5], we may suppose that $\mu^{(m)} \in\left(E^{\prime}\right)_{\mathcal{U}}$. Let $x^{(n)}=\left(x_{i}^{(n)}\right)$ and $\mu^{(n)}=\left(\mu_{i}^{(n)}\right)$ for each $n$.

Let $\lambda \in E^{\prime}$ and $x \in E$ be such that $\langle\lambda, x\rangle=1$. For each $n \geq 1$, define

$$
T_{n}=\mu^{(n)} \otimes x=\left(\mu_{i}^{(n)} \otimes x\right) \in(\mathcal{A}(E))_{\mathcal{U}}, \quad S_{n}=\lambda \otimes x^{(n)}=\left(\lambda \otimes x_{i}^{(n)}\right) \in(\mathcal{A}(E))_{\mathcal{U}} .
$$

Define $\Lambda \in(\mathcal{A}(E))_{\mathcal{U}}^{\prime}$ by

$$
\langle\Lambda, R\rangle=\lim _{i \rightarrow \mathcal{U}}\left\langle\lambda, R_{i}(x)\right\rangle \quad\left(R=\left(R_{i}\right) \in(\mathcal{A}(E))_{\mathcal{U}}\right) .
$$

It is hence easy to see that

$$
\left\langle\Lambda, T_{n} S_{m}\right\rangle=\left\langle\mu^{(n)}, x^{(m)}\right\rangle,
$$

from which it follows that $\Lambda$ is not weakly almost periodic, as required.

Notice that in the above proof, $\Lambda$ is a member of $\mathcal{A}(E)^{\prime}$, where we naturally embed $\mathcal{A}(E)^{\prime}$ into $(\mathcal{A}(E))_{\mathcal{U}}^{\prime}$. Hence, when $E$ is not super-reflexive, $(\mathcal{A}(E))_{\mathcal{U}}$ fails to be Arens regular is this rather strong sense.

An alternative way to see the above is the following. For a Banach space $E$, we can regards $(\mathcal{A}(E))_{\mathcal{U}}$ as a subalgebra of $\mathcal{B}\left((E)_{\mathcal{U}}\right)$ in the usual way. It is then easy to see that $\mathcal{A}\left((E)_{\mathcal{U}}\right)$ is contained in $(\mathcal{A}(E))_{\mathcal{U}}$, and so if $(\mathcal{A}(E))_{\mathcal{U}}$ is Arens regular, so is $\mathcal{A}\left((E)_{\mathcal{U}}\right)$, and hence, as mentioned above, $(E)_{\mathcal{U}}$ must be reflexive.

Combining the above results, we might be tempted to make the following conjecture: a Banach algebra $\mathcal{A}$ is super Arens regular if and only if $\mathcal{A}$ is isomorphic to a subalgebra of $\mathcal{B}(E)$ for some super-reflexive Banach space $E$. In [34, Young showed that a Banach algebra $\mathcal{A}$ is isomorphic to a subalgebra of $\mathcal{B}(E)$ for a reflexive $E$ if and only if $\operatorname{WAP}\left(\mathcal{A}^{\prime}\right)$ approximately norms $\mathcal{A}$, that is, for some $\delta>0$,

$$
\|a\| \geq \delta \sup \left\{|\langle\mu, a\rangle|: \mu \in \operatorname{WAP}\left(\mathcal{A}^{\prime}\right),\|\mu\| \leq 1\right\} \quad(a \in \mathcal{A}) .
$$

In particular, Arens regular Banach algebras are even isometric to closed subalgebras of $\mathcal{B}(E)$ for reflexive $E$. The key tool which Young uses is that of interpolation spaces, although this wasn't recognised at the time (compare Kaijser's work in [19]). However, it is not clear how interpolation spaces and ultrapowers interact; just because an ultrapower $(\mathcal{A})_{\mathcal{U}}$ is isomorphic to a subalgebra of $\mathcal{B}(E)$ does not seem to imply that $E$ need to be ultrapower.

\subsection{Ultrapowers of dual Banach algebras}

Surprisingly, defining ultrapowers of dual Banach algebras is not as straight forward as for von Neumann algebras: we have to take account of Arens regularity. 
Recall that a dual Banach algebra is a Banach algebra $\mathcal{A}$ which is the dual of a Banach space, say $\mathcal{A}=\mathcal{A}_{*}^{\prime}$, such that the product on $\mathcal{A}$ is separately weak ${ }^{*}$-continuous. The canonical example is a von Neumann algebra, in which case the predual $\mathcal{A}_{*}$ is isometrically unique. In general, there may be a choice of $\mathcal{A}_{*}$, so we shall write $\left(\mathcal{A}, \mathcal{A}_{*}\right)$ to indicate the predual. See [31] or [8] for general further information.

By analogy with the von Neumann case, the natural way to define an ultrapower of $\mathcal{A}$ is to form the Banach space ultrapower $\left(\mathcal{A}_{*}\right)_{\mathcal{U}}$, and then to extend the product from $(\mathcal{A})_{\mathcal{U}}$ to the dual $\operatorname{space}\left(\mathcal{A}_{*}\right)_{\mathcal{U}}^{\prime}$.

Proposition 3.5. Let $\left(\mathcal{A}, \mathcal{A}_{*}\right)$ be a dual Banach algebra, and let $\mathcal{U}$ be an ultrafilter on an index set I. Let $\mathfrak{A}_{*}=\left(\mathcal{A}_{*}\right)_{\mathcal{U}}$ and $\mathfrak{A}=\mathfrak{A}_{*}^{\prime}$. The following are equivalent:

1. There is a product on $\mathfrak{A}$ extending the product on $(\mathcal{A})_{\mathcal{U}}$ and turning $\left(\mathfrak{A}, \mathfrak{A}_{*}\right)$ into a dual Banach algebra;

2. If we identify $\left(\mathcal{A}_{*}\right)_{\mathcal{U}}$ with a subspace of $(\mathcal{A})_{\mathcal{U}}^{\prime}$, we have that $\left(\mathcal{A}_{*}\right)_{\mathcal{U}} \subseteq \operatorname{WAP}\left((\mathcal{A})_{\mathcal{U}}^{\prime}\right)$.

Proof. Notice that as $(\mathcal{A})_{\mathcal{U}}$ is weak*-dense in $\mathfrak{A}$, any product making $\left(\mathfrak{A}, \mathfrak{A}_{*}\right)$ into a dual Banach algebra, and which extends the product on $(\mathcal{A})_{\mathcal{U}}$, must be unique. If (1) holds then it is an easy calculation (see [8, Section 2]) that $\mathfrak{A}_{*} \subseteq \operatorname{WAP}\left(\mathfrak{A}^{\prime}\right)$. Condition (2) is immediate from this.

Conversely, notice that $\mathfrak{A}=\left(\mathcal{A}_{*}\right)_{\mathcal{U}}$ is an $(\mathcal{A})_{\mathcal{U}}$-bimodule, and so $\mathfrak{A}^{\prime}$ is also an $(\mathcal{A})_{\mathcal{U}}$-bimodule. It is obvious that this bimodule structure extends the product on $(\mathcal{A})_{\mathcal{U}}$. We can hence extend this bimodule structure to a bilinear map on $\mathfrak{A}$, either by extending on the left, or on the right, by weak*-continuity. Let us check that these give the same result. Let $a, b \in \mathfrak{A}$, so by [16, Section 7], there exist bounded nets $\left(a_{\alpha}\right)$ and $\left(b_{\alpha}\right)$ in $(\mathcal{A})_{\mathcal{U}}$, tending to $a$ and $b$ respectively. For $\mu \in \mathfrak{A}_{*}$, we see that

$$
\begin{aligned}
\lim _{\alpha}\left\langle a_{\alpha} \cdot b, \mu\right\rangle & =\lim _{\alpha}\left\langle b, \mu \cdot a_{\alpha}\right\rangle=\lim _{\alpha} \lim _{\beta}\left\langle b_{\beta}, \mu \cdot a_{\alpha}\right\rangle=\lim _{\alpha} \lim _{\beta}\left\langle a_{\alpha} b_{\beta}, \mu\right\rangle \\
& =\lim _{\beta} \lim _{\alpha}\left\langle a_{\alpha} b_{\beta}, \mu\right\rangle=\lim _{\beta}\left\langle a \cdot b_{\beta}, \mu\right\rangle .
\end{aligned}
$$

We can swap the order of the limits, as $\mu \in \operatorname{WAP}\left((\mathcal{A})_{\mathcal{U}}^{\prime}\right)$. The construction of this product is very similar to the construction of the Arens products, and checking that our product on $\mathfrak{A}$ is associative is similar to the analogous calculation for the Arens products.

Finally, we show that $\left(\mathfrak{A}, \mathfrak{A}_{*}\right)$ is a dual Banach algebra, for which it suffices to check that $\mathfrak{A}_{*}$ is an $\mathfrak{A}$-submodule of $\mathfrak{A}^{\prime}$. Let $\mu \in \mathfrak{A}_{*}$ and $a \in \mathfrak{A}$, and suppose that $a \cdot \mu \notin \mathfrak{A}_{*} \subseteq \mathfrak{A}^{\prime}$, so there exists $\Phi \in \mathfrak{A}^{\prime \prime}$ annihilating $\mathfrak{A}_{*}$ and with $\langle\Phi, a \cdot \mu\rangle=1$. Let $\left(b_{\alpha}\right)$ be a bounded net in $\mathfrak{A}$ tending to $\Phi$ weak* in $\mathfrak{A}^{\prime \prime}$. For each $\alpha$, let $\left(c_{\alpha, \beta}\right)$ be a bounded net in $(\mathcal{A})_{\mathcal{U}}$ tending to $b_{\alpha}$ weak* in $\mathfrak{A}$. Let $\left(c_{\gamma}\right)$ be a bounded net in $(\mathcal{A})_{\mathcal{U}}$ tending to $a$ in the weak*-topology on $\mathfrak{A}$. Then we see that

$$
\begin{aligned}
1 & =\langle\Phi, a \cdot \mu\rangle=\lim _{\alpha}\left\langle a \cdot \mu, b_{\alpha}\right\rangle=\lim _{\alpha}\left\langle b_{\alpha} a, \mu\right\rangle=\lim _{\alpha} \lim _{\beta}\left\langle c_{\alpha, \beta} a, \mu\right\rangle=\lim _{\alpha} \lim _{\beta}\left\langle a, \mu \cdot c_{\alpha, \beta}\right\rangle \\
& =\lim _{\alpha} \lim _{\beta} \lim _{\gamma}\left\langle c_{\gamma}, \mu \cdot c_{\alpha, \beta}\right\rangle=\lim _{\alpha} \lim _{\beta} \lim _{\gamma}\left\langle c_{\alpha, \beta} c_{\gamma}, \mu\right\rangle=\lim _{\gamma} \lim _{\alpha} \lim _{\beta}\left\langle c_{\alpha, \beta} c_{\gamma}, \mu\right\rangle \\
& =\lim _{\gamma} \lim _{\alpha}\left\langle b_{\alpha}, c_{\gamma} \cdot \mu\right\rangle=0,
\end{aligned}
$$

a contradiction. Again, we use that $\mu \in \operatorname{WAP}\left((\mathcal{A})_{\mathcal{U}}^{\prime}\right)$ to allow us to swap the order of limits. Hence $a \cdot \mu \in \mathfrak{A}_{*}$, and similarly $\mu \cdot a \in \mathfrak{A}_{*}$, as required.

Notice that if $\mathcal{A}$ is super Arens regular, then certainly condition (2) above always holds.

Proposition 3.6. Let $\left(\mathcal{A}, \mathcal{A}_{*}\right)$ be a dual Banach algebra, and suppose that for all ultrafilters $\mathcal{U}$, we have that $\left(\mathcal{A}_{*}\right)_{\mathcal{U}} \subseteq \operatorname{WAP}\left((\mathcal{A})_{\mathcal{U}}^{\prime}\right)$. Then every even dual of $\mathcal{A}$ is Arens regular. 
Proof. Firstly we show that $\mathcal{A}$ is Arens regular. Let $\mu \in \mathcal{A}^{\prime}$ and let $\left(a_{n}\right)$ and $\left(b_{m}\right)$ be bounded sequences in $\mathcal{A}$ with the repeated $\operatorname{limits}_{n} \lim _{n} \lim _{m}\left\langle\mu, a_{n} b_{m}\right\rangle$ and $\lim _{m} \lim _{n}\left\langle\mu, a_{n} b_{m}\right\rangle$ existing. By [16. Proposition 6.7], for a suitable ultrafilter $\mathcal{U}$, there exists $\left(\mu_{i}\right) \in\left(\mathcal{A}_{*}\right)_{\mathcal{U}}$ with

$$
\lim _{i \rightarrow \mathcal{U}}\left\langle a, \mu_{i}\right\rangle=\langle\mu, a\rangle \quad(a \in \mathcal{A}) .
$$

As $\left(\mathcal{A}_{*}\right)_{\mathcal{U}} \subseteq \operatorname{WAP}\left((\mathcal{A})_{\mathcal{U}}^{\prime}\right)$, we have that $\left(\mu_{i}\right) \in \operatorname{WAP}\left((\mathcal{A})_{\mathcal{U}}^{\prime}\right)$, and so

$$
\begin{aligned}
\lim _{n} \lim _{m}\left\langle\mu, a_{n} b_{m}\right\rangle & =\lim _{n} \lim _{m} \lim _{i \rightarrow \mathcal{U}}\left\langle a_{n} b_{m}, \mu_{i}\right\rangle=\lim _{n} \lim _{m}\left\langle\left(a_{n}\right)\left(b_{m}\right),\left(\mu_{i}\right)\right\rangle \\
& =\lim _{m} \lim _{n}\left\langle\left(a_{n}\right)\left(b_{m}\right),\left(\mu_{i}\right)\right\rangle=\lim _{m} \lim _{n}\left\langle\mu, a_{n} b_{m}\right\rangle,
\end{aligned}
$$

as required.

Let $\Lambda \in \mathcal{A}^{\prime \prime \prime}$ and let $\left(\Phi_{n}\right)$ and $\left(\Psi_{m}\right)$ be bounded sequences in $\mathcal{A}^{\prime \prime}$ with the repeated limits $\lim _{n} \lim _{m}\left\langle\Lambda, \Phi_{n} \Psi_{m}\right\rangle$ and $\lim _{m} \lim _{n}\left\langle\Lambda, \Phi_{n} \Psi_{m}\right\rangle$ existing. For an ultrapower $(\mathcal{A})_{\mathcal{U}}$, define a map $\sigma_{\mathcal{U}}:(\mathcal{A})_{\mathcal{U}} \rightarrow \mathcal{A}^{\prime \prime}$ by

$$
\left\langle\sigma_{\mathcal{U}}(a), \mu\right\rangle=\lim _{i \rightarrow \mathcal{U}}\left\langle\mu, a_{i}\right\rangle \quad\left(a=\left(a_{i}\right) \in(\mathcal{A})_{\mathcal{U}}\right)
$$

As $\mathcal{A}$ is Arens regular, by the main result of [7], there exists an ultrafilter $\mathcal{U}$ on an index set $I$, and a map $K: \mathcal{A}^{\prime \prime} \rightarrow(\mathcal{A})_{\mathcal{U}}$, such that $\sigma_{\mathcal{U}} \circ K$ is the identity on $\mathcal{A}^{\prime \prime}$, and

$$
\left\langle\sigma_{\mathcal{U}}(K(\Phi) K(\Psi)), \mu\right\rangle=\langle\Phi \square \Psi, \mu\rangle \quad\left(\mu \in \mathcal{A}^{\prime}, \Phi, \Psi \in \mathcal{A}^{\prime \prime}\right) .
$$

There exists an ultrafilter $\mathcal{V}$ on an index set $J$ such that $\sigma_{\mathcal{V}}:\left(\mathcal{A}^{\prime}\right)_{\mathcal{V}} \rightarrow \mathcal{A}^{\prime \prime \prime}$ is surjective.

We define (see the end of Section 7 in [16]) the ultrafilter $\mathcal{U} \times \mathcal{V}$ on $I \times J$ by, for $A \subseteq I \times J$, setting $A \in \mathcal{U} \times \mathcal{V}$ if and only if

$$
\{i \in I:\{j \in J:(i, j) \in A\} \in \mathcal{V}\} \in \mathcal{U}
$$

Then, for a family $\left(x_{i, j}\right)_{i \in I, j \in J}$ in a compact Hausdorff space $X$, we have that

$$
\lim _{j \rightarrow \mathcal{V}} \lim _{i \rightarrow \mathcal{U}} x_{i, j}=\lim _{(i, j) \rightarrow \mathcal{U} \times \mathcal{V}} x_{i, j}
$$

For each $n$ let $K\left(\Phi_{n}\right)=\left(a_{i}^{(n)}\right) \in(\mathcal{A})_{\mathcal{U}}$, and let $K\left(\Psi_{n}\right)=\left(b_{i}^{(n)}\right) \in(\mathcal{A})_{\mathcal{U}}$. Let $\left(\mu_{j}\right) \in\left(\mathcal{A}^{\prime}\right)_{\mathcal{V}}$ be such that $\sigma_{\mathcal{V}}\left(\left(\mu_{j}\right)\right)=\Lambda$. We then see that, as $\left(\mu_{j}\right) \in \operatorname{WAP}\left((\mathcal{A})_{\mathcal{U} \times \mathcal{V}}^{\prime}\right)$,

$$
\begin{aligned}
\lim _{n} \lim _{m}\left\langle\Lambda, \Phi_{n} \Psi_{m}\right\rangle & =\lim _{n} \lim _{m} \lim _{j \rightarrow \mathcal{V}}\left\langle\Phi_{n} \Psi_{m}, \mu_{j}\right\rangle=\lim _{n} \lim _{m} \lim _{j \rightarrow \mathcal{V}} \lim _{i \rightarrow \mathcal{U}}\left\langle\mu_{j}, a_{i}^{(n)} b_{i}^{(m)}\right\rangle \\
& =\lim _{n} \lim _{m} \lim _{(i, j) \rightarrow \mathcal{U} \times \mathcal{V}}\left\langle\mu_{j}, a_{i}^{(n)} b_{i}^{(m)}\right\rangle \\
& =\lim _{m} \lim _{n} \lim _{(i, j) \rightarrow \mathcal{U} \times \mathcal{V}}\left\langle\mu_{j}, a_{i}^{(n)} b_{i}^{(m)}\right\rangle=\lim _{m} \lim _{n}\left\langle\Lambda, \Phi_{n} \Psi_{m}\right\rangle .
\end{aligned}
$$

Hence $\mathcal{A}^{\prime \prime}$ is Arens regular.

Repeating this argument allows us to show that every even dual of $\mathcal{A}$ is Arens regular, as claimed.

Again, it would be interesting to know if, when every even dual of a Banach algebra $\mathcal{A}$ is Arens regular, we have $\mathcal{A}$ is super Arens regular? In conclusion, we see that our approach to ultrapowers of dual Banach algebras requires a rather strong condition on the underlying algebra, indeed, in practice, we need $\mathcal{A}$ to be a subalgebra of $\mathcal{B}(E)$ for a super-reflexive Banach space $E$. 


\section{Tensor products of ultrapowers}

We shall now sketch the basics of the theory of tensor products of Banach spaces. We refer the reader to the books [32] or [12] for introductory treatments of this material, or to the book [11] for further information.

For Banach spaces $E$ and $F$, let $E \otimes F$ be the algebraic tensor product of $E$ and $F$. We define the projective tensor norm by

$$
\|\tau\|_{\pi}=\inf \left\{\sum_{i=1}^{n}\left\|x_{i}\right\|\left\|y_{i}\right\|: \tau=\sum_{i=1}^{n} x_{i} \otimes y_{i}\right\} \quad(\tau \in E \otimes F) .
$$

The completion of $E \otimes F$ with respect to $\|\cdot\|_{\pi}$ is $E \widehat{\otimes} F$, the projective tensor product of $E$ and $F$. $E \widehat{\otimes} F$ has the universal property that if $T: E \times F \rightarrow G$ is a bounded bilinear map to a Banach space $G$, then there is a unique bounded linear map $\widehat{T}: E \widehat{\otimes} F \rightarrow G$ such that $\widehat{T}(x \otimes y)=T(x, y)$ for $x \in E$ and $y \in F$. Every member $\tau \in E \widehat{\otimes} F$ can be written as an absolutely convergent sum $\tau=\sum_{i=1}^{\infty} x_{i} \otimes y_{i}$, for some sequences $\left(x_{i}\right) \subseteq E$ and $\left(y_{i}\right) \subseteq F$.

Let $\mathcal{F}(E, F)$ be the space of finite-rank operators from $E$ to $F$, and let $\mathcal{A}(E, F)$ be the space of approximable operators from $E$ to $F$, the norm closure of $\mathcal{F}(E, F)$ in $\mathcal{B}(E, F)$. We can embed $E \otimes F$ into $\mathcal{F}\left(E^{\prime}, F\right)$ by

$$
\left(\sum_{i=1}^{n} x_{i} \otimes y_{i}\right): \mu \mapsto \sum_{i=1}^{n}\left\langle\mu, x_{i}\right\rangle y_{i} \quad\left(\mu \in E^{\prime}\right)
$$

This induces the injective tensor norm $\|\cdot\|_{\epsilon}$ on $E \otimes F$, whose completion is $E \check{\otimes} F$. In particular, we can identify $\mathcal{A}(E, F)$ with $E^{\prime} \ddot{\otimes} F$.

We shall say that norm $\|\cdot\|$ on $E \otimes F$ is a reasonable crossnorm when:

1. $\|x \otimes y\|=\|x\|\|y\|$ for $x \in E$ and $y \in F$;

2. for $\mu \in E^{\prime}$ and $\lambda \in F^{\prime}$, define $\mu \otimes \lambda: E \otimes F \rightarrow \mathbb{C}$ by $\langle\mu \otimes \lambda, x \otimes y\rangle=\langle\mu, x\rangle\langle\lambda, y\rangle$ and linearity. Then the norm of $\mu \otimes \lambda$, with respect to $\|\cdot\|$, is $\|\mu\|\|\lambda\|$.

Suppose that for each pair of Banach spaces $(E, F)$, we have an assignment of a reasonable crossnorm $\|\cdot\|$ on $E \otimes F$. Then this assignment is a uniform crossnorm when given pairs $\left(E_{1}, F_{1}\right)$ and $\left(E_{2}, F_{2}\right)$ of Banach spaces, for $T \in \mathcal{B}\left(E_{1}, E_{2}\right), S \in \mathcal{B}\left(F_{1}, F_{2}\right)$, we have that $\|T \otimes S\| \leq\|T\|\|S\|$ where we treat $T \otimes S$ as a linear map $E_{1} \widehat{\otimes} F_{1} \rightarrow E_{2} \widehat{\otimes} F_{2}$ given by

$$
(T \otimes S)(x \otimes y)=T(x) \otimes S(y) \quad\left(x \otimes y \in E_{1} \otimes F_{1}\right),
$$

and linearity. Then $\|\cdot\|_{\pi}$ and $\|\cdot\|_{\epsilon}$ are uniform crossnorms.

The projective tensor product is projective in the sense that if $T$ and $S$ are quotient maps (also called metric surjections) then so is $T \otimes S: E_{1} \widehat{\otimes} F_{1} \rightarrow E_{2} \widehat{\otimes} F_{2}$. Similarly, the injective tensor product is injective in that, when $T$ and $S$ are isometries, then so is $T \otimes S: E_{1} \check{\otimes} F_{1} \rightarrow E_{2} \check{\otimes} F_{2}$. In general, the projective tensor norm is not injective, and the injective tensor norm is not projective. A useful exception to this is that the map $\kappa_{E} \otimes \mathrm{id}: E \widehat{\otimes} F \rightarrow E^{\prime \prime} \widehat{\otimes} F$ is always an isometry onto its range.

We identify the dual of $E \widehat{\otimes} F$ with $\mathcal{B}\left(E, F^{\prime}\right)$ by

$$
\langle T, x \otimes y\rangle=\langle T(x), y\rangle \quad\left(T \in \mathcal{B}\left(E, F^{\prime}\right), x \otimes y \in E \widehat{\otimes} F\right),
$$

and linearity and continuity. In particular, $E^{\prime} \check{\otimes} F^{\prime}=\mathcal{A}\left(E, F^{\prime}\right)$ isometrically embeds into $(E \widehat{\otimes} F)^{\prime}$. When one of $E$ or $F$ is finite-dimensional, we have equality, $(E \widehat{\otimes} F)^{\prime}=E^{\prime} \check{\otimes} F^{\prime}$. 
As the map $E \widehat{\otimes} F \rightarrow E \check{\otimes} F$ is norm-decreasing with dense range, we see that the adjoint $(E \check{\otimes} F)^{\prime} \rightarrow(E \widehat{\otimes} F)^{\prime}=\mathcal{B}\left(E, F^{\prime}\right)$ is norm-decreasing and injective. We hence identify $(E \check{\otimes} F)^{\prime}$ with a space of operators $E \rightarrow F^{\prime}$, the integral operators $\mathcal{I}\left(E, F^{\prime}\right)$, and we give $\mathcal{I}\left(E, F^{\prime}\right)$ the dual norm $\|\cdot\|_{\mathcal{I}}$, so that $\mathcal{I}\left(E, F^{\prime}\right)=(E \check{\otimes} F)^{\prime}$. We have a norm-decreasing map $E^{\prime} \widehat{\otimes} F^{\prime} \rightarrow \mathcal{I}\left(E, F^{\prime}\right)$. It is quite a subtle issue as to when this map is bounded below, an isometry, or when it is surjective. See [32] or [11, Section 16] for further details. However, if one of $E$ or $F$ is finite-dimensional, then $(E \check{\otimes} F)^{\prime}=\mathcal{I}\left(E, F^{\prime}\right)=E^{\prime} \widehat{\otimes} F^{\prime}$.

We say that a Banach space $E$ has the approximation property when the canonical map $E^{\prime} \widehat{\otimes} E \rightarrow$ $E^{\prime} \check{\otimes} E=\mathcal{A}(E)$ is injective. See [32, Chapter 4] or [12, Chapter VIII] for further details. For Banach spaces $E$ and $F$ with the approximation property, we can hence identify $E^{\prime} \widehat{\otimes} F$ as a space of operators from $E$ to $F$, called the nuclear operators, $\mathcal{N}(E, F)$. In general, $\mathcal{N}(E, F)$ is merely a quotient of $E^{\prime} \widehat{\otimes} F$, and we always give $\mathcal{N}(E, F)$ the quotient norm.

\subsection{Ultrapowers}

Let $M$ be a finite-dimensional Banach space and let $\mathcal{U}$ be an ultrafilter. By taking a basis, it is easy to see that $(M)_{\mathcal{U}}=M$. It is shown in [16, Lemma 7.4], that

$$
(M \check{\otimes} E)_{\mathcal{U}}=M \check{\otimes}(E)_{\mathcal{U}} \quad, \quad(M \widehat{\otimes} E)_{\mathcal{U}}=M \widehat{\otimes}(E)_{\mathcal{U}}
$$

for every Banach space $E$, and every finite-dimensional $M$, with equality of norms.

For infinite-dimensional Banach spaces, these equalities are no longer necessarily true. However, we can make some useful statements.

Let $E$ and $F$ be Banach spaces. There is a canonical map $\psi_{0}:(E)_{\mathcal{U}} \widehat{\otimes}(F)_{\mathcal{U}} \rightarrow(E \widehat{\otimes} F)_{\mathcal{U}}$, defined using the tensorial property of $\widehat{\otimes}$. Firstly we define $\psi_{0}:(E)_{\mathcal{U}} \times(F)_{\mathcal{U}} \rightarrow(E \widehat{\otimes} F)_{\mathcal{U}}$ by

$$
\psi_{0}(x, y)=\left(x_{i} \otimes y_{i}\right) \quad\left(x=\left(x_{i}\right) \in(E)_{\mathcal{U}}, y=\left(y_{i}\right) \in(F)_{\mathcal{U}}\right)
$$

Then we have

$$
\left\|\left(x_{i} \otimes y_{i}\right)\right\|=\lim _{i \rightarrow \mathcal{U}}\left\|x_{i} \otimes y_{i}\right\|_{\pi}=\lim _{i \rightarrow \mathcal{U}}\left\|x_{i}\right\|\left\|y_{i}\right\|=\left(\lim _{i \rightarrow \mathcal{U}}\left\|x_{i}\right\|\right)\left(\lim _{i \rightarrow \mathcal{U}}\left\|y_{i}\right\|\right)=\|x\|\|y\|,
$$

so that $\psi_{0}$ is well-defined, and is a norm-decreasing bilinear map. Thus $\psi_{0}$ extends to a normdecreasing map $\psi_{0}:(E)_{\mathcal{U}} \widehat{\otimes}(F)_{\mathcal{U}} \rightarrow(E \widehat{\otimes} F)_{\mathcal{U}}$. For $\tau \in(E)_{\mathcal{U}} \otimes(F)_{\mathcal{U}}$, choose a representative $\tau=\sum_{k=1}^{n} x_{k} \otimes y_{k}$. Let, for each $k, x_{k}=\left(x_{i}^{(k)}\right) \in(E)_{\mathcal{U}}$ and $y_{k}=\left(y_{i}^{(k)}\right) \in(E)_{\mathcal{U}}$. Then we see that

$$
\psi_{0}(\tau)=\left(\sum_{k=1}^{n} x_{i}^{(k)} \otimes y_{i}^{(k)}\right)_{i \in I} \in(E \widehat{\otimes} F)_{\mathcal{U}}
$$

Proposition 4.1. Let $E$ and $F$ be Banach spaces, let $\mathcal{U}$ be an ultrafilter on an index set $I$, and let $\tau \in(E \widehat{\otimes} F)_{\mathcal{U}}$. Then the following are equivalent:

1. for some sequence $\left(\alpha_{n}\right)$ of positive reals with $\sum_{n} \alpha_{n}<\infty, \tau=\left(\tau_{i}\right)$ admits a representation of the form

$$
\tau_{i}=\sum_{k=1}^{\infty} x_{k}^{(i)} \otimes y_{k}^{(i)} \in E \widehat{\otimes} F \quad(i \in I),
$$

where, for each $i$ and $k$, we have that $\left\|x_{k}^{(i)}\right\|\left\|y_{k}^{(i)}\right\| \leq \alpha_{k}$;

2. $\tau$ lies in the image of $\psi_{0}$. 
Proof. Suppose that (1) holds. By rescaling, we may suppose that $\left\|x_{k}^{(i)}\right\|=\left\|y_{k}^{(i)}\right\| \leq \alpha_{k}^{1 / 2}$ for each $i \in I$ and $k \geq 1$. For each $k \geq 1$, let

$$
x_{k}=\left(x_{k}^{(i)}\right) \in(E)_{\mathcal{U}}, \quad y_{k}=\left(y_{k}^{(i)}\right) \in(F)_{\mathcal{U}}
$$

so that $\left\|x_{k}\right\| \leq \alpha_{k}^{1 / 2}$ and $\left\|y_{k}\right\| \leq \alpha_{k}^{1 / 2}$. We can hence let

$$
\sigma=\sum_{k=1}^{\infty} x_{k} \otimes y_{k} \in(E)_{\mathcal{U}} \widehat{\otimes}(F)_{\mathcal{U}}
$$

with $\pi(\sigma) \leq \sum_{k} \alpha_{k}$. Let $\sigma_{n}=\sum_{k=1}^{n} x_{k} \otimes y_{k}$ so that $\sigma_{n} \rightarrow \sigma$ in $(E)_{\mathcal{U}} \widehat{\otimes}(F)_{\mathcal{U}}$. Then

$$
\begin{aligned}
\lim _{n \rightarrow \infty}\left\|\psi_{0}\left(\sigma_{n}\right)-\tau\right\| & =\lim _{n \rightarrow \infty} \lim _{i \rightarrow \mathcal{U}}\left\|\sum_{k=1}^{n} x_{k}^{(i)} \otimes y_{k}^{(i)}-\tau_{i}\right\|_{\pi} \\
& \leq \lim _{n \rightarrow \infty} \lim _{i \rightarrow \mathcal{U}} \sum_{k=n+1}^{\infty}\left\|x_{k}^{(i)}\right\|\left\|y_{k}^{(i)}\right\| \leq \lim _{n \rightarrow \infty} \sum_{k=n+1}^{\infty} \alpha_{k}=0
\end{aligned}
$$

so that $\psi_{0}(\sigma)=\tau$, as required.

Conversely, suppose that $\tau=\psi_{0}(\sigma)$ for

$$
\sigma=\sum_{k=1}^{\infty} x_{k} \otimes y_{k} \in(E)_{\mathcal{U}} \widehat{\otimes}(F)_{\mathcal{U}}
$$

with $\sum_{k=1}^{\infty}\left\|x_{k}\right\|\left\|y_{k}\right\|<\infty$. Then we let $\alpha_{k}=\left\|x_{k}\right\|\left\|y_{k}\right\|$ and pick representatives $x_{k}=\left(x_{k}^{(i)}\right) \in(E)_{\mathcal{U}}$ and $y_{k}=\left(y_{k}^{(i)}\right) \in(E)_{\mathcal{U}}$, with $\left\|x_{k}\right\|=\left\|x_{k}^{(i)}\right\|$ and $\left\|y_{k}\right\|=\left\|y_{k}^{(i)}\right\|$ for each $k$ and $i$. For each $i \in I$, let $\tau_{i}=\sum_{k=1}^{\infty} x_{k}^{(i)} \otimes y_{k}^{(i)}$. Let $\sigma_{n}=\sum_{k=1}^{n} x_{k} \otimes y_{k}$, so that $\tau=\lim _{n \rightarrow \infty} \psi_{0}\left(\sigma_{n}\right)$. Thus, for each $n$,

$$
\left\|\left(\tau_{i}\right)-\psi_{0}\left(\sigma_{n}\right)\right\|=\lim _{i \rightarrow \mathcal{U}}\left\|\tau_{i}-\sum_{k=1}^{n} x_{k}^{(i)} \otimes y_{k}^{(i)}\right\|_{\pi} \leq \lim _{i \rightarrow \mathcal{U}} \sum_{k=n+1}^{\infty}\left\|x_{k}^{(i)}\right\|\left\|y_{k}^{(i)}\right\|=\sum_{k=n+1}^{\infty} \alpha_{k} .
$$

Hence, letting $n \rightarrow \infty,\left(\tau_{i}\right)=\tau$ as required.

Let $\mathcal{A}$ be a Banach algebra, and let $E$ be a left- $\mathcal{A}$-module. Then an ultrapower $(E)_{\mathcal{U}}$ becomes a left- $\mathcal{A}$-module in the obvious way. When $F$ is a right- $\mathcal{A}$-module, we have that $E \widehat{\otimes} F$ is an $\mathcal{A}$-bimodule for the module actions

$$
a \cdot(x \otimes y)=a \cdot x \otimes y, \quad(x \otimes y) \cdot a=x \otimes y \cdot a \quad(a \in \mathcal{A}, x \otimes y \in E \widehat{\otimes} F) .
$$

Hence an ultrapower $(E \widehat{\otimes} F)_{\mathcal{U}}$ is also an $\mathcal{A}$-bimodule. Similarly, $(E)_{\mathcal{U}} \widehat{\otimes}(F)_{\mathcal{U}}$ is an $\mathcal{A}$-bimodule. It is a simple check to see that $\psi_{0}$ is an $\mathcal{A}$-bimodule homomorphism.

Similarly, it is easily checked that $(E)_{\mathcal{U}}$ is a left- $(\mathcal{A})_{\mathcal{U}}$-module, $(F)_{\mathcal{U}}$ is a right- $(\mathcal{A})_{\mathcal{U}}$-module, and both $(E \widehat{\otimes} F)_{\mathcal{U}}$ and $(E)_{\mathcal{U}} \widehat{\otimes}(F)_{\mathcal{U}}$ are $(\mathcal{A})_{\mathcal{U}}$-bimodules. We can check that $\psi_{0}$ is also an $(\mathcal{A})_{\mathcal{U}}$-bimodule homomorphism.

In general, it seems that $\psi_{0}$ is rarely, if ever, surjective when $E$ and $F$ are infinite-dimensional. We now present an argument for Hilbert spaces that is motivated by a counter-example communicated to us by Charles Read. We first recall the Schmidt representation theorem (see, for example, the treatment given in [26]). 
Theorem 4.2. Let $H$ and $K$ be Hilbert spaces, and $T \in \mathcal{A}(H, K)$. Then there exist orthonormal sequences $\left(h_{n}\right)$ and $\left(k_{n}\right)$ in $H$ and $K$, respectively, and a sequence of positive numbers $\left(s_{n}\right) \in c_{0}(\mathbb{N})$ with $s_{1} \geq s_{2} \geq \cdots$ such that

$$
T(x)=\sum_{n=1}^{\infty} s_{n}\left[x, h_{n}\right] k_{n} \quad(x \in H),
$$

where $[\cdot, \cdot]$ is the inner-product on $H$.

Here and henceforth, we allow orthonormal sequences to be eventually zero.

For a Hilbert space $H$ and $x \in H$, we define a linear functional $x^{*}$ on $H$ by $y \mapsto[y, x]$. The Riesz Theorem shows that every linear functional arises in this way. It is clear that if the sequence $\left(s_{n}\right)$ above satisfies $\sum_{n} s_{n}<\infty$, then $T$ will be nuclear, and hence identified with a member of $H \widehat{\otimes} K$ (as $H$ and $K$ have the approximation property) with $\|T\|_{\pi} \leq \sum_{n} s_{n}$.

Lemma 4.3. If $T \in H \widehat{\otimes} K$, then the sequence $\left(s_{n}\right)$ arising from the Schmidt representation of $T$ satisfies $\|T\|_{\pi}=\sum_{n} s_{n}$.

Proof. For $\epsilon>0$, let $T=\sum_{n} u_{n}^{*} \otimes v_{n}$ with $\sum_{n}\left\|u_{n}\right\|\left\|v_{n}\right\|<\|T\|_{\pi}+\epsilon$. Then, by the Schmidt representation, we have also that $T=\sum_{n} s_{n} h_{n}^{*} \otimes k_{n}$, say. Then

$$
\begin{aligned}
\sum_{n} s_{n} & =\sum_{n}\left[T\left(h_{n}\right), k_{n}\right]=\sum_{n} \sum_{m}\left[h_{n}, u_{m}\right]\left[v_{m}, k_{n}\right] \\
& \leq \sum_{m}\left(\sum_{n}\left|\left[h_{n}, u_{m}\right]\right|^{2}\right)^{1 / 2}\left(\sum_{n}\left|\left[v_{m}, k_{n}\right]\right|^{2}\right)^{1 / 2} \\
& \leq \sum_{m}\left\|u_{m}\right\|\left\|v_{m}\right\|<\|T\|_{\pi}+\epsilon,
\end{aligned}
$$

as $\left(h_{n}\right)$ and $\left(k_{n}\right)$ are orthonormal sequences. As $\epsilon>0$ was arbitrary, we are done.

Notice that this proof shows that, for $T \in H \widehat{\otimes} K$, we have that

$$
\|T\|_{\pi}=\sup \left\{\sum_{n}\left|\left[T\left(e_{n}\right), f_{n}\right]\right|:\left(e_{n}\right) \text { and }\left(f_{n}\right) \text { are orthonormal sequences in } H \text { and } K\right\} .
$$

We now recall the notion (see [26, Chapter 11]) of approximation numbers. Let $E$ and $F$ be Banach spaces, and let $T \in \mathcal{B}(E, F)$. The $n$th approximation number of $T$, for $n \geq 1$, is

$$
a_{n}(T)=\inf \{\|T-S\|: S \in \mathcal{F}(E, F), \operatorname{rank}(S)<n\} .
$$

Proposition 4.4. Let $H$ and $K$ be Hilbert spaces, and let $T \in \mathcal{A}(H, K)$ have a Schmidt representation $T=\sum_{n} s_{n} h_{n}^{*} \otimes k_{n}$. Then, if $s_{1} \geq s_{2} \geq \cdots$, then $s_{n}=a_{n}(T)$ for each $n \geq 1$.

Proof. See [26, Section 11.3].

Proposition 4.5. Let $H$ and $K$ be Hilbert spaces, and let $\mathcal{U}$ be a ultrafilter on an index set $I$. Then each $\tau \in(H \widehat{\otimes} K)_{\mathcal{U}}$ in the image of $\psi_{0}$ admits a representation of the form $\tau=\left(\tau_{i}\right)$ with

$$
\tau_{i}=\sum_{n} s_{n} h_{n, i}^{*} \otimes k_{n, i} \in H \widehat{\otimes} K \quad(i \in I),
$$

where $\left(s_{n}\right)$ is a sequence of positive reals with $\sum_{n} s_{n}<\infty$ and, for each $i,\left(h_{n, i}\right)$ and $\left(k_{n, i}\right)$ are orthonormal sequences in $H$ and $K$ respectively. 
Proof. Let $\tau=\psi_{0}(\sigma)$ where

$$
\sigma=\sum_{n} s_{n} e_{n}^{*} \otimes f_{n}
$$

where $\left(e_{n}\right)$ and $\left(f_{n}\right)$ are orthonormal sequences in $(H)_{\mathcal{U}}$ and $(K)_{\mathcal{U}}$ respectively. Pick representatives $e_{n}=\left(e_{n, i}\right)_{i \in I}$ and $f_{n}=\left(f_{n, i}\right)_{i \in I}$, so that

$$
\delta_{n, m}=\lim _{i \rightarrow \mathcal{U}}\left[e_{n, i}, e_{m, i}\right]=\lim _{i \rightarrow \mathcal{U}}\left[f_{n, i}, f_{m, i}\right] \quad(n, m \geq 1) .
$$

For each $i$, apply the Gram-Schmidt orthonormalisation process to $\left(e_{n, i}\right)$ to yield $\left(h_{n, i}\right)$, where we allow $h_{n, i}$ to be zero for sufficiently large $n$; do the same to $\left(f_{n, i}\right)$ to yield $\left(k_{n, i}\right)$. For each $n \geq 1$, as $h_{n, i}$ depends only upon $\left\{e_{m, i}: m \leq n\right\}$ and $\left\{\left[e_{m, i}, e_{r, i}\right]: m, r \leq n\right\}$, we can verify that

$$
\lim _{i \rightarrow \mathcal{U}}\left\|h_{n, i}-e_{n, i}\right\|=0 \quad(n \geq 1),
$$

and similarly for $f_{n, i}$. Let $h_{n}=\left(h_{n, i}\right)_{i \in I} \in(H)_{\mathcal{U}}$ and $k_{n}=\left(k_{n, i}\right)_{i \in I} \in(H)_{\mathcal{U}}$, so that $h_{n}=e_{n}$ and $k_{n}=f_{n}$. Thus

$$
\tau=\psi_{0}(\sigma)=\left(\sum_{n} s_{n} h_{n, i}^{*} \otimes k_{n, i}\right)_{i \in I} \in(H \widehat{\otimes} K)_{\mathcal{U}},
$$

as required.

Theorem 4.6. Let $H$ and $K$ be Hilbert spaces, and let $\mathcal{U}$ be a countably incomplete ultrafilter on an index set $I$. Then $\psi_{0}:(H)_{\mathcal{U}} \widehat{\otimes}(K)_{\mathcal{U}} \rightarrow(H \widehat{\otimes} K)_{\mathcal{U}}$ does not have dense range.

Proof. We first consider the case when $I=\mathbb{N}$ and $\mathcal{U}$ is a non-principal ultrafilter on $\mathbb{N}$. Let $\left(e_{n}\right)$ and $\left(f_{n}\right)$ be orthonormal sequences in $H$ and $K$, respectively. For each $n \geq 1$, let

$$
\tau_{n}=n^{-1} \sum_{j=1}^{n} e_{j}^{*} \otimes f_{j} \in H \widehat{\otimes} K,
$$

and let $\tau=\left(\tau_{n}\right) \in(H \widehat{\otimes} K)_{\mathcal{U}}$. Let $\sigma \in(H \widehat{\otimes} K)_{\mathcal{U}}$ be in the image of $\psi_{0}$, so that $\sigma$ has a representation as above,

$$
\sigma=\left(\sigma_{k}\right)=\left(\sum_{n} s_{n} h_{n, k}^{*} \otimes k_{n, k}\right)_{k \in \mathbb{N}} \in(H \widehat{\otimes} K)_{\mathcal{U}} .
$$

Pick $\epsilon>0$, and choose $N$ such that $\sum_{n>N} s_{n}<\epsilon$. Then

$$
\begin{aligned}
\lim _{k \rightarrow \mathcal{U}} \pi\left(\tau_{k}-\sigma_{k}\right) & >\lim _{k \rightarrow \mathcal{U}} \pi\left(\tau_{k}-\sum_{n=1}^{N} s_{n} h_{n, k}^{*} \otimes k_{n, k}\right)-\epsilon \\
& \geq \lim _{k \rightarrow \mathcal{U}} \inf \left\{\pi\left(\tau_{k}-v\right): v \in \mathcal{F}(H, K), \operatorname{rank}(v) \leq N\right\}-\epsilon \\
& =\lim _{k \rightarrow \mathcal{U}} \inf \left\{\sum_{m \geq 1} a_{m}\left(\tau_{k}-v\right): v \in \mathcal{F}(H, K), \operatorname{rank}(v) \leq N\right\}-\epsilon,
\end{aligned}
$$

by an application of Proposition 4.4. Now, for $v \in \mathcal{F}(H, K)$ with $\operatorname{rank}(v) \leq N$, it is clear that $a_{m}\left(\tau_{k}-v\right) \geq a_{N+m}\left(\tau_{k}\right)$, so that

$$
\lim _{k \rightarrow \mathcal{U}} \pi\left(\tau_{k}-\sigma_{k}\right)>\lim _{k \rightarrow \mathcal{U}}(k-N) k^{-1}-\epsilon=1-\epsilon .
$$

As $\epsilon>0$ and $\sigma$ were arbitrary, we see that $\tau$ is distance 1 (as $\pi(\tau)=1)$ from the image of $\psi_{0}$.

A standard argument allows us to adapt this proof to the case when $\mathcal{U}$ is an arbitrary countably incomplete ultrafilter on an index set $I$ (compare with the proofs of Theorem 6.3 or Proposition 7.1 in [16]). 
The above seems to rely very heavily upon certain special features of Hilbert spaces, as did the original counter-example due to C. J. Read. It would be interesting to know how $(E)_{\mathcal{U}} \widehat{\otimes}(F)_{\mathcal{U}}$ and $(E \widehat{\otimes} F)_{\mathcal{U}}$ relate for other classes of Banach spaces.

For the following, we refer the reader to [16, Section 9], where Heinrich gives a description of when $(E)_{\mathcal{U}}$ has the approximation property. In particular, the following are equivalent: $(E)_{\mathcal{U}}$ has the approximation property for all $\mathcal{U} ; E$ has the uniform approximation property; $(E)_{\mathcal{U}}$ has the approximation property for some non-principal $\mathcal{U}$ on a countable index set. Notice that, by [16, Theorem 3.3], $\left(L^{p}(\nu)\right)_{\mathcal{U}}$ has the approximation property for any measure $\nu, 1 \leq p \leq \infty$, and any $\mathcal{U}$.

Proposition 4.7. Let $E$ and $F$ be Banach spaces such that $F$ is super-reflexive. Let $\mathcal{U}$ be an ultrafilter such that $(F)_{\mathcal{U}}$ has the approximation property. Then $\psi_{0}:(E)_{\mathcal{U}} \widehat{\otimes}(F)_{\mathcal{U}} \rightarrow(E \widehat{\otimes} F)_{\mathcal{U}}$ is an isometry onto its range.

Proof. As $(F)_{\mathcal{U}}$ is reflexive and $(F)_{\mathcal{U}}$ has the approximation property, we have

$$
\mathcal{A}\left((E)_{\mathcal{U}},\left(F^{\prime}\right)_{\mathcal{U}}\right)^{\prime}=\left((E)_{\mathcal{U}}^{\prime} \check{\otimes}\left(F^{\prime}\right)_{\mathcal{U}}\right)^{\prime}=\mathcal{I}\left((E)_{\mathcal{U}}^{\prime},(F)_{\mathcal{U}}\right)=(E)_{\mathcal{U}}^{\prime \prime} \widehat{\otimes}(F)_{\mathcal{U}}
$$

See [32, Section 5.3] for details. As the map $\kappa_{(E)_{\mathcal{U}}} \otimes \mathrm{id}:(E)_{\mathcal{U}} \widehat{\otimes}(F)_{\mathcal{U}} \rightarrow(E)_{\mathcal{U}}^{\prime \prime} \widehat{\otimes}(F)_{\mathcal{U}}$ is an isometry onto its range, we see that

$$
\|\tau\|_{\pi}=\sup \left\{|\langle\tau, S\rangle|: S \in \mathcal{F}\left((E)_{\mathcal{U}},\left(F^{\prime}\right)_{\mathcal{U}}\right),\|S\| \leq 1\right\} \quad\left(\tau \in(E)_{\mathcal{U}} \widehat{\otimes}(F)_{\mathcal{U}}\right)
$$

In the following, for a Banach space $X$, we write $\operatorname{FIN}(X)$ for the collection of finite-dimensional subspaces of $X$. Fix $\tau \in(E)_{\mathcal{U}} \otimes(F)_{\mathcal{U}}$. Let $\tau=\sum_{k=1}^{n} y^{(k)} \otimes z^{(k)}$ and let $N=\operatorname{lin}\left\{y^{(k)}: 1 \leq k \leq n\right\} \in$ $\operatorname{FIN}\left((E)_{\mathcal{U}}\right)$. For each $k$, let $y^{(k)}=\left(y_{i}^{(k)}\right)$ and $z^{(k)}=\left(z_{i}^{(k)}\right)$ where $\left\|y^{(k)}\right\|=\left\|y_{i}^{(k)}\right\|$ and $\left\|z^{(k)}\right\|=\left\|z_{i}^{(k)}\right\|$ for each $i$. Thus

$$
\psi_{0}(\tau)=\left(\tau_{i}\right)=\left(\sum_{k=1}^{n} y_{i}^{(k)} \otimes z_{i}^{(k)}\right)_{i \in I} \in(E \widehat{\otimes} F)_{\mathcal{U}}
$$

Choose $\epsilon>0$ and let $S \in \mathcal{F}\left((E)_{\mathcal{U}},\left(F^{\prime}\right)_{\mathcal{U}}\right)$ be such that $\|S\| \leq 1$ and $|\langle\tau, S\rangle|>\|\tau\|_{\pi}-\epsilon$. Let $M=S\left((E)_{\mathcal{U}}\right) \in \mathrm{FIN}\left(\left(F^{\prime}\right)_{\mathcal{U}}\right)$ have a basis $\left\{x^{(1)}, \ldots, x^{(m)}\right\}$ where $x^{(k)}=\left(x_{i}^{(k)}\right) \in(E)_{\mathcal{U}}^{\prime}=\left(E^{\prime}\right)_{\mathcal{U}}$ for each $k$. Following the proof of [16, Proposition 6.2], let $M_{i}=\operatorname{lin}\left\{x_{i}^{(k)}: 1 \leq k \leq m\right\} \in \mathrm{FIN}\left(F^{\prime}\right)$ and $T_{i}: M \rightarrow M_{i}$ be defined by $T_{i}\left(x^{(k)}\right)=x_{i}^{(k)}$. Then, for some $I_{0} \in \mathcal{U}, T_{i}$ is a $(1+\epsilon)$-isomorphism for each $i \in I_{0}$.

We can write $S=\sum_{k=1}^{m} \mu^{(k)} \otimes x^{(k)}$ for some $\left(\mu^{(k)}\right)_{k=1}^{m} \subseteq(E)_{\mathcal{U}}^{\prime}$. Let $P=\operatorname{lin}\left\{\mu^{(k)}: 1 \leq k \leq m\right\} \in$ $\operatorname{FIN}\left((E)_{\mathcal{U}}^{\prime}\right)$. By [16, Theorem 7.3], there exists a $(1+\epsilon)$-isomorphism onto its range $T: P \rightarrow\left(E^{\prime}\right) \mathcal{U}$ such that

$$
\left\langle T\left(\mu^{(k)}\right), z\right\rangle=\left\langle\mu^{(k)}, z\right\rangle \quad(1 \leq k \leq m, z \in N) .
$$

For each $k$, let $T\left(\mu^{(k)}\right)=\left(\mu_{i}^{(k)}\right) \in\left(E^{\prime}\right) \mathcal{U}$. Then let $Q=T(P)$, let $Q_{i}=\operatorname{lin}\left\{\mu_{i}^{(k)}: 1 \leq k \leq m\right\} \in$ $\operatorname{FIN}\left(E^{\prime}\right)$ and let $R_{i}: Q \rightarrow Q_{i}$ be given by $R_{i}\left(T\left(\mu^{(k)}\right)\right)=\mu_{i}^{(k)}$. Again, there exists $I_{1} \in \mathcal{U}$ such that $R_{i}$ is a $(1+\epsilon)$-isomorphism for each $i \in I_{1}$.

For each $i \in I_{0} \cap I_{1} \in \mathcal{U}$, let

$$
S_{i}=\sum_{k=1}^{m} R_{i} T\left(\mu^{(k)}\right) \otimes T_{i}\left(x^{(k)}\right)=\left(R_{i} T \otimes T_{i}\right) S \in F^{\prime} \otimes E^{\prime}=\mathcal{F}\left(F, E^{\prime}\right),
$$


so that $\left\|S_{i}\right\| \leq\left\|R_{i}\right\|\|T\|\left\|T_{i}\right\|\|S\| \leq(1+\epsilon)^{3}$. Then we have

$$
\begin{aligned}
\langle\tau, S\rangle & =\sum_{j=1}^{n}\left\langle S\left(y^{(j)}\right), z^{(j)}\right\rangle=\sum_{j=1}^{n} \sum_{k=1}^{m}\left\langle\mu^{(k)}, y^{(j)}\right\rangle\left\langle x^{(k)}, z^{(j)}\right\rangle \\
& =\sum_{j=1}^{n} \sum_{k=1}^{m}\left\langle T\left(\mu^{(k)}\right), y^{(j)}\right\rangle\left\langle x^{(k)}, z^{(j)}\right\rangle=\sum_{j=1}^{n} \sum_{k=1}^{m} \lim _{i \rightarrow \mathcal{U}}\left\langle\mu_{i}^{(k)}, y_{i}^{(j)}\right\rangle\left\langle x_{i}^{(k)}, z_{i}^{(j)}\right\rangle \\
& =\lim _{i \rightarrow \mathcal{U}} \sum_{j=1}^{n} \sum_{k=1}^{m}\left\langle R_{i} T\left(\mu^{(k)}\right), y_{i}^{(j)}\right\rangle\left\langle T_{i}\left(x^{(k)}\right), z_{i}^{(j)}\right\rangle=\lim _{i \rightarrow \mathcal{U}}\left\langle S_{i}, \tau_{i}\right\rangle .
\end{aligned}
$$

As $\psi_{0}$ is norm-decreasing, we conclude that

$$
\|\tau\|_{\pi}-\epsilon<\lim _{i \rightarrow \mathcal{U}}\left|\left\langle S_{i}, \tau_{i}\right\rangle\right| \leq \lim _{i \rightarrow \mathcal{U}}\left\|S_{i}\right\| \pi\left(\tau_{i}\right) \leq(1+\epsilon)^{3}\left\|\psi_{0}(\tau)\right\| \leq(1+\epsilon)^{3}\|\tau\|_{\pi}
$$

As $\epsilon>0$ was arbitrary, we conclude that $\psi_{0}$ is an isometry onto its range.

\section{$5 \quad$ Ultra-amenability}

Let $\mathcal{A}$ be a Banach algebra and $E$ be a Banach $\mathcal{A}$-bimodule. Then a derivation $d: \mathcal{A} \rightarrow E$ is a linear map such that $d(a b)=a \cdot d(b)+d(a) \cdot b$ for $a, b \in \mathcal{A}$. All the derivations which we shall consider will be continuous. Let $x \in E$ and define $\delta_{x}: \mathcal{A} \rightarrow E$ by $\delta_{x}(a)=a \cdot x-x \cdot a$. Then $\delta_{x}$ is a derivation, termed an inner derivation.

A Banach algebra $\mathcal{A}$ is contractible or super-amenable if every derivation from $\mathcal{A}$ to a Banach $\mathcal{A}$-bimodule is inner. A contractible Banach algebra is unital, and it is conjectured that a Banach algebra $\mathcal{A}$ is contractible only when $\mathcal{A}$ is finite-dimensional. This is true for $\mathrm{C}^{*}$-algebras (indeed, for closed subalgebras of $\mathcal{B}(H)$ for a Hilbert space $H$, see [25]) and for $\mathcal{B}(E)$ when $E$ has, for example, the approximation property (see [30, Section 4.1]).

A Banach algebra $\mathcal{A}$ is amenable if every derivation from $\mathcal{A}$ to a dual Banach $\mathcal{A}$-bimodule is inner. For example, commutative, unital $\mathrm{C}^{*}$-algebras (that is, $C(K)$ spaces with pointwise product) are amenable; group algebras $L^{1}(G)$ are amenable if and only if the locally compact group $G$ is amenable (see [30] for these and further results).

Let $\mathcal{A}$ be a Banach algebra, and turn $\mathcal{A} \widehat{\otimes} \mathcal{A}$ into a Banach $\mathcal{A}$-bimodule by

$$
a \cdot(b \otimes c)=a b \otimes c, \quad(b \otimes c) \cdot a=b \otimes c a \quad(a \in \mathcal{A}, b \otimes c \in \mathcal{A} \widehat{\otimes} \mathcal{A}),
$$

and linearity and continuity. Define the product map $\Delta_{\mathcal{A}}: \mathcal{A} \widehat{\otimes} \mathcal{A} \rightarrow \mathcal{A}$ by $\Delta_{\mathcal{A}}(a \otimes b)=a b$. The following result, due to Johnson, can be found in [30, Section 2.2].

Theorem 5.1. Let $\mathcal{A}$ be a Banach algebra. Then $\mathcal{A}$ is contractible if and only if there exists a diagonal $\tau \in \mathcal{A} \widehat{\otimes} \mathcal{A} ;$ that is, $a \cdot \tau=\tau \cdot a$ and $\Delta_{\mathcal{A}}(\tau) a=$ a for each $a \in \mathcal{A}$.

Similarly, $\mathcal{A}$ is amenable if and only if, for some $C>0$, for each $\epsilon>0$ and $a_{1}, \ldots, a_{n} \in \mathcal{A}$, there exists $\tau \in \mathcal{A} \widehat{\otimes} \mathcal{A}$ such that $\|\tau\|_{\pi} \leq C,\left\|a_{i} \cdot \tau-\tau \cdot a_{i}\right\|_{\pi}<\epsilon$ and $\left\|\Delta_{\mathcal{A}}(\tau) a_{i}-a_{i}\right\|<\epsilon$ for $1 \leq i \leq n$.

We shall say that $\mathcal{A}$ is $C$-amenable if the above holds for the constant $C>0$.

We define a Banach algebra $\mathcal{A}$ to be ultra-amenable if every ultrapower of $\mathcal{A}$ is amenable. It would be more natural, in light of terms like "super-reflexive", to call this super-amenable, but this term is already used by Runde in [30] for contractible algebras (as "contractible" has multiple meanings as well!) We see immediately that unital, commutative $\mathrm{C}^{*}$-algebras are ultra-amenable. 
Proposition 5.2. Let $\mathcal{A}$ be a Banach algebra, and let $\mathcal{I}$ be a closed ideal in $\mathcal{A}$. When $\mathcal{A}$ is ultraamenable, $\mathcal{A} / \mathcal{I}$ is ultra-amenable. If $\mathcal{I}$ and $\mathcal{A} / \mathcal{I}$ are ultra-amenable, then so is $\mathcal{A}$. Furthermore, when $\mathcal{A}$ is ultra-amenable, $\mathcal{I}$ is ultra-amenable if and only if $\mathcal{I}$ has a bounded approximate identity.

Proof. Let $\mathcal{U}$ be an ultrafilter. Then the quotient map $\mathcal{A} \rightarrow \mathcal{A} / \mathcal{I}$ induces a quotient map $(\mathcal{A})_{\mathcal{U}} \rightarrow$ $(\mathcal{A} / \mathcal{I})_{\mathcal{U}}$, and so $(\mathcal{A} / \mathcal{I})_{\mathcal{U}}$ is amenable by [30, Corollary 2.3.2]. Indeed, as in the Banach space case (compare [16, Proposition 6.5]), we can identify $(\mathcal{A} / \mathcal{I})_{\mathcal{U}}$ with $(\mathcal{A})_{\mathcal{U}} /(\mathcal{I})_{\mathcal{U}}$. Hence if $(\mathcal{A} / \mathcal{I})_{\mathcal{U}}$ and $(\mathcal{I})_{\mathcal{U}}$ are both amenable, then so is $(\mathcal{A})_{\mathcal{U}}$ by [30, Theorem 2.3.10].

As $(\mathcal{A})_{\mathcal{U}}$ is amenable and $(\mathcal{I})_{\mathcal{U}}$ is an ideal in $(\mathcal{A})_{\mathcal{U}}$, by [30, Theorem 2.3.7], $(\mathcal{I})_{\mathcal{U}}$ is amenable if and only if it has a bounded approximate identity. By Proposition 2.2, this is equivalent to $\mathcal{I}$ having a bounded approximate identity, as required.

\subsection{Diagonal-like constructions}

Instead of working with the definition of amenability, it is common to work with approximate or virtual diagonals; see [30, Chapter 2] (and Theorem [5.1 above). In this section, we provide a similar characterisation of ultra-amenability.

Definition 5.3. Let $\mathcal{A}$ be a Banach algebra, and let $n \geq 1$. Let $S_{n}(\mathcal{A})$ be the collection of subsets of size $n$ of the unit sphere of $\mathcal{A}$.

Let $C>0, \epsilon>0$ and $n \geq 1$. For $A \subseteq S_{n}(\mathcal{A})$, we say that $A \in D_{n}(\mathcal{A}, C, \epsilon)$ when there exists a sequence of positive reals $\left(\alpha_{k}\right)$ with $\sum_{k} \alpha_{k} \leq C$, and such that for each $F \in A$, we have that there exists

$$
\tau=\sum_{k=1}^{\infty} a_{k} \otimes b_{k} \in \mathcal{A} \widehat{\otimes} \mathcal{A}
$$

with

$$
\|a \cdot \tau-\tau \cdot a\|_{\pi} \leq \epsilon, \quad\left\|\Delta_{\mathcal{A}}(\tau) a-a\right\| \leq \epsilon \quad(a \in F),
$$

and with $\left\|a_{k}\right\|\left\|b_{k}\right\| \leq \alpha_{k}$ for each $k$.

Proposition 5.4. Let $\mathcal{A}$ be a Banach algebra, let $\mathcal{U}$ be an ultrafilter on an index set $I$, and let $C>0$ be a constant. Then the following are equivalent:

1. $(\mathcal{A})_{\mathcal{U}}$ is $C$-amenable;

2. for each $n \geq 1$, each $\epsilon>0$, and each map $\gamma: I \rightarrow S_{n}(\mathcal{A})$, there exists a sequence of positive reals $\left(\alpha_{j}\right)$, with $\sum_{j} \alpha_{j} \leq C$, and there exists $B \in \mathcal{U}$ such that for each $i \in B$, there exists $\tau=\sum_{j=1}^{\infty} b_{j} \otimes c_{j} \in \mathcal{A} \widehat{\otimes} \mathcal{A}$ with

$$
\|a \cdot \tau-\tau \cdot a\|_{\pi}<\epsilon, \quad\left\|\Delta_{\mathcal{A}}(\tau) a-a\right\|<\epsilon \quad(a \in \gamma(i)),
$$

and with $\left\|b_{j}\right\|\left\|c_{j}\right\| \leq \alpha_{j}$ for $j \geq 1$.

3. for each $n \geq 1$, each $\epsilon>0$, and each map $\gamma: I \rightarrow S_{n}(\mathcal{A})$, there exists $A \in D_{n}(\mathcal{A}, C, \epsilon)$ with $\gamma^{-1}(A) \in \mathcal{U}$.

Proof. By definition, $(\mathcal{A})_{\mathcal{U}}$ is amenable if and only if there exists $C>0$ such that, for each $\epsilon>0$ and each $a^{(1)}, \cdots, a^{(n)} \in(\mathcal{A})_{\mathcal{U}}$, there exists $\tau \in(\mathcal{A})_{\mathcal{U}} \widehat{\otimes}(\mathcal{A})_{\mathcal{U}}$ such that

$$
\left\|a^{(k)} \cdot \tau-\tau \cdot a^{(k)}\right\|_{\pi}<\epsilon, \quad\left\|\Delta_{(\mathcal{A})_{\mathcal{U}}}(\tau) a^{(k)}-a^{(k)}\right\|<\epsilon \quad(1 \leq k \leq n),
$$

and with $\|\tau\|_{\pi} \leq C$. Now, we may suppose that the $a^{(k)}$ are distinct, and, by a perturbation argument, that $a_{i}^{(j)} \neq a_{i}^{(k)}$ for each $i \in I$ and $j \neq k$, while $\left\|a_{i}^{(k)}\right\|=\left\|a^{(k)}\right\|$ for each $i \in I$. As we are 
free to vary $\epsilon>0$, it is enough to consider the case when $\left\|a^{(k)}\right\|=1$ for each $k$. Thus the choice of the family $\left\{a^{(k)}: 1 \leq k \leq n\right\}$ corresponds to a choice of a map $\gamma: I \rightarrow S_{n}(\mathcal{A})$, together with some ordering.

As explained before, $\psi_{0}:(\mathcal{A})_{\mathcal{U}} \widehat{\otimes}(\mathcal{A})_{\mathcal{U}} \rightarrow(\mathcal{A} \widehat{\otimes} \mathcal{A})_{\mathcal{U}}$ is both an $\mathcal{A}$-bimodule homomorphism, and an $(\mathcal{A})_{\mathcal{U}}$-bimodule homomorphism. Furthermore, the following diagram commutes

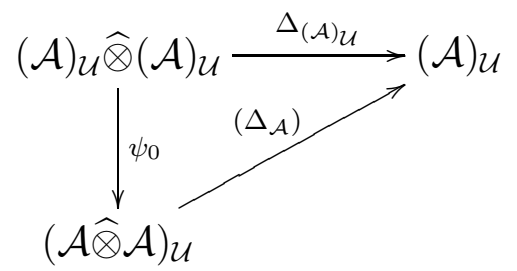

Thus let $\psi_{0}(\tau)=\left(\tau_{i}\right)_{i \in I}$, so that our conditions upon $\tau$ imply that

$$
\lim _{i \rightarrow \mathcal{U}}\left\|a_{i}^{(k)} \cdot \tau_{i}-\tau_{i} \cdot a_{i}^{(k)}\right\|_{\pi}<\epsilon, \quad \lim _{i \rightarrow \mathcal{U}}\left\|\Delta_{\mathcal{A}}\left(\tau_{i}\right) a_{i}^{(k)}-a_{i}^{(k)}\right\|<\epsilon \quad(1 \leq k \leq n) .
$$

By definition, this is so if and only if there exists $B \in \mathcal{U}$ with

$$
\left\|a \cdot \tau_{i}-\tau_{i} \cdot a\right\|_{\pi}<\epsilon, \quad\left\|\Delta_{\mathcal{A}}\left(\tau_{i}\right) a-a\right\|<\epsilon \quad(i \in B, a \in \gamma(i)) .
$$

With reference to Proposition 4.1, we have that (1) implies (2).

Conversely, we simply apply Proposition 4.1 to build $\tau \in(\mathcal{A})_{\mathcal{U}} \widehat{\otimes}(\mathcal{A})_{\mathcal{U}}$ out of the family $\left(\tau_{i}\right)$, where the condition upon the $\left(\tau_{i}\right)$ implies that we can work in the image of $\psi_{0}$.

Finally, it is easy to see that (2) and (3) are equivalent.

Corollary 5.5. Let $\mathcal{A}$ be a Banach algebra. If $\mathcal{A}$ is contractible, then $\mathcal{A}$ is ultra-amenable. If $\mathcal{A}$ is ultra-amenable, $\mathcal{A}$ is amenable.

By considering commutative $\mathrm{C}^{*}$-algebras, we see that being contractible is strictly stronger than being ultra-amenable. We shall see below that being ultra-amenable is also strictly stronger than being amenable.

Theorem 5.6. Let $\mathcal{A}$ be a Banach algebra. The following are equivalent:

1. $\mathcal{A}$ is ultra-amenable;

2. there exists a constant $C>0$ such that for every $n \geq 1$ and $\epsilon>0$, there exists a finite partition $S_{n}(\mathcal{A})=A_{1} \cup \cdots \cup A_{k}$ with each $A_{i} \in D_{n}(\mathcal{A}, \bar{C}, \epsilon)$.

Proof. Suppose that (2) holds. We shall verify condition (3) of Proposition 5.4, which will show that (11) holds. If $\mathcal{U}$ is an ultrafilter on an index set $I$ and $\gamma: I \rightarrow S_{n}(\mathcal{A})$ is a map, then it is easily checked that

$$
\gamma^{*}(\mathcal{U}):=\left\{A \subseteq S_{n}(\mathcal{A}): \gamma^{-1}(A) \in \mathcal{U}\right\}
$$

is an ultrafilter on $S_{n}(\mathcal{A})$. Thus there exists some $k$ with $A_{k} \in \gamma^{*}(\mathcal{U})$, that is, $\gamma^{-1}\left(A_{k}\right) \in \mathcal{U}$, as required.

Suppose that (1) holds. We first introduce a little notation. For an ultrafilter $\mathcal{U}$ on an index set $I$, for a property $p$ of elements of $I$, we write

$$
\forall_{\mathcal{U}} i, p(i) \Leftrightarrow\{i \in I: p(i) \text { holds }\} \in \mathcal{U} .
$$

For each $n \geq 1$, let $\mathcal{U}_{n}$ be an ultrafilter on $S_{n}(\mathcal{A})$. For each $n$, define an arbitrary injection $\iota_{n}: S_{n}(\mathcal{A}) \rightarrow S_{n+1}(\mathcal{A})$. Let $I$ be the collection of sequences $\left(F_{n}\right)$ where $F_{n} \in S_{n}(\mathcal{A})$ for each $n$, and for some $N>0$ (depending on the sequence), we have that $F_{n+1}=\iota_{n}\left(F_{n}\right)$ for $n \geq N$. 
Loosely speaking, $I$ is the collection of eventually constant sequences in $\bigcup_{n} S_{n}(\mathcal{A})$. Let $\mathcal{U}$ be a non-principal ultrafilter on $\mathcal{N}$. We define an ultrafilter $\mathcal{V}$ on $I$ by setting $K \in \mathcal{V}$ if and only if

$$
\forall_{\mathcal{U}} n, \forall_{\mathcal{U}_{1}} F_{1}, \cdots \forall_{\mathcal{U}_{n}} F_{n},\left(F_{1}, F_{2}, \cdots, F_{n}, F_{n}, \cdots\right) \in K
$$

It is an easy check that $\mathcal{V}$ is an ultrafilter. Suppose that $(\mathcal{A})_{\mathcal{V}}$ is $C$-amenable for some constant $C>0$.

Let $n \geq 1$, and define a map $\gamma: I \rightarrow S_{n}(\mathcal{A})$ by $\gamma\left(\left(F_{k}\right)\right)=F_{n}$ for $\left(F_{k}\right) \in I$. Hence by Proposition 5.4, for each $\epsilon>0$, there exists $A \in D_{n}(\mathcal{A}, C, \epsilon)$ with $\gamma^{-1}(A) \in \mathcal{V}$. By the definition of $\mathcal{V}$, this means that

$$
\forall_{\mathcal{U}} m, \forall_{\mathcal{U}_{1}} F_{1}, \cdots \forall_{\mathcal{U}_{m}} F_{m}, \gamma\left(F_{1}, F_{2}, \cdots, F_{m}, F_{m}, \cdots\right) \in A
$$

Hence, for some $m>n$, by the definition of $\gamma$, we see that

$$
\forall_{\mathcal{U}_{1}} F_{1}, \cdots \forall_{\mathcal{U}_{m}} F_{m}, F_{n} \in A
$$

that is, $\forall_{\mathcal{U}_{n}} F_{n}, F_{n} \in A$, which is simply the statement that $A \in \mathcal{U}_{n}$. In conclusion, for each $n \geq 1$ and $\epsilon>0$, there exists some member of $D_{n}(\mathcal{A}, C, \epsilon)$ in $\mathcal{U}_{n}$.

If condition (2) does not hold, then for some $n \geq 1$ and $\epsilon>0$, we have that there is no finite cover of $S_{n}(\mathcal{A})$ by members of $D_{n}(\mathcal{A}, C, \epsilon)$. In particular, if $\mathcal{F}=\left\{S_{n}(\mathcal{A}) \backslash A: A \in D_{n}(\mathcal{A}, C, \epsilon)\right\}$ then, as no finite intersection of members of $\mathcal{F}$ is empty, there exists an ultrafilter $\mathcal{U}_{n}$ containing $\mathcal{F}$. However, by the previous paragraph, we know that $\mathcal{U}_{n} \cap D_{n}(\mathcal{A}, C, \epsilon)$ is non-empty, which gives a contradiction, as required.

\section{$5.2 \quad \mathrm{C}^{*}$-algebras}

By throwing a lot of machinery at the problem, we can rather easily settle the question of when a $\mathrm{C}^{*}$-algebra is ultra-amenable. In [20], there is a throwaway comment in the proof of Theorem 2.5 that, for a $\mathrm{C}^{*}$-algebra $\mathcal{A}$, every ultrapower $(\mathcal{A})_{\mathcal{U}}$ is amenable if and only if $\ell^{\infty}(\mathcal{A}, I)$ is amenable for all index sets $I$. We do not see why this is "obvious" however, as in general $(\mathcal{A})_{\mathcal{U}}$ is much smaller than $\ell^{\infty}(\mathcal{A}, I)$. The following proof avoids this issue, and uses no more machinery than [20] does.

Theorem 5.7. Let $\mathcal{A}$ be a $C^{*}$-algebra. Then the following are equivalent:

1. $\mathcal{A}$ is ultra-amenable;

2. $\mathcal{A}^{\prime \prime}$ is amenable;

3. $\mathcal{A}$ is the finite-direct sum of algebras of the form $C_{0}(K) \otimes \mathbb{M}_{n}$ for some integer $n$ and some locally compact Hausdorff space $K$.

Proof. By [30, Corollary 6.4.28] and [30, Corollary 6.4.29], we have that when a $\mathrm{C}^{*}$-algebra is amenable, it has the (metric) approximation property. Hence, if (1) holds, then for any ultrafilter $\mathcal{U}$, we have that $(\mathcal{A})_{\mathcal{U}}$ has the approximation property. For a suitable choice of $\mathcal{U}$, we have that $\mathcal{A}^{\prime \prime}$ is isometric to a complemented subspace of $(\mathcal{A})_{\mathcal{U}}$ (see [16, Proposition 6.7]). As the approximation property clearly drops to complemented subspaces, we conclude that $\mathcal{A}^{\prime \prime}$ has the approximation property. By [30, Theorem 6.1.7] and [30, Remark 6.1.9], this implies that $\mathcal{A}^{\prime \prime}$ is amenable, giving (2).

When (2) holds, by [30, Theorem 6.1.7], we know that $\mathcal{A}^{\prime \prime}$ has the form specified in (3), but with $K$ compact, and such that $C_{0}(K)=C(K)$ is a dual space (so that $K$ is actually a hyperstonian space). Suppose that $\mathcal{A}^{\prime \prime}$ were isomorphic to $C(K)$ for some hyperstonian compact space $X$. Then $\mathcal{A}$ is commutative, and so is isomorphic to $C_{0}(L)$, for some locally compact space $L$. Then note 
that the bidual of $C_{0}(L) \otimes \mathbb{M}_{n}$ is isomorphic to $C(K) \otimes \mathbb{M}_{n}$, which is isomorphic to $\mathcal{A}^{\prime \prime}$. It is now clear that $\mathcal{A}$ must be isomorphic to $C_{0}(L) \otimes \mathbb{M}_{n}$, showing (3).

Finally, when (3) holds, it is clear that $\mathcal{A}$ is ultra-amenable, giving (11).

It seems unlikely that (1) and (2) are equivalent for a general Banach algebra $\mathcal{A}$, but we do not currently have a counter-example. It would, of course, be nice to be able to prove the above without using so much machinery, even for certain classes of $\mathrm{C}^{*}$-algebra.

\subsection{Group algebras}

Let $G$ be a locally compact group, and form the Banach algebra $L^{1}(G)$. See [3, Section 3.3] or [22, Section 1.9], for example, for details about this class of algebras. We shall make use of the concept of the almost periodic, or Bohr, compactification for a group $G$. However, it makes sense for us to develop these ideas first for general Banach algebras.

Above, for a Banach algebra $\mathcal{A}$, we defined the space of weakly almost periodic functionals of $\mathcal{A}$, denoted by $\operatorname{WAP}\left(\mathcal{A}^{\prime}\right)$. If we insist that the map $L_{\mu}$, for $\mu \in \mathcal{A}^{\prime}$,

$$
L_{\mu}: \mathcal{A} \rightarrow \mathcal{A}^{\prime} ; \quad a \mapsto a \cdot \mu \quad(a \in \mathcal{A})
$$

is compact, and not just weakly-compact, we arrive at the definition of an almost periodic functional, denoted by $\mu \in \operatorname{AP}\left(\mathcal{A}^{\prime}\right)$. Clearly $\operatorname{AP}\left(\mathcal{A}^{\prime}\right) \subseteq \operatorname{WAP}\left(\mathcal{A}^{\prime}\right)$, and it is easy to show that $\operatorname{AP}\left(\mathcal{A}^{\prime}\right)$ is a closed submodule of $\mathcal{A}^{\prime}$.

Let $\mathcal{A}$ be a Banach algebra, and let $\mathcal{U}$ be an ultrafilter. Then we can define

$$
\sigma_{\mathrm{AP}}:(\mathcal{A})_{\mathcal{U}} \rightarrow \operatorname{AP}\left(\mathcal{A}^{\prime}\right)^{\prime}, \quad\left\langle\sigma_{\mathrm{AP}}\left(\left(a_{i}\right)\right), \mu\right\rangle=\lim _{i \rightarrow \mathcal{U}}\left\langle\mu, a_{i}\right\rangle \quad\left(\mu \in \operatorname{AP}\left(\mathcal{A}^{\prime}\right),\left(a_{i}\right) \in(\mathcal{A})_{\mathcal{U}}\right)
$$

It is clear that $\sigma_{\mathrm{AP}}$ is norm-decreasing, and for suitable $\mathcal{U}, \sigma_{\mathrm{AP}}$ is a surjection (compare [16, Proposition 6.7]). As remarked upon in [7, Section 5], $\sigma_{\mathrm{AP}}$ is easily seen to be an algebra homomorphism.

Proposition 5.8. Let $\mathcal{A}$ be an ultra-amenable Banach algebra. Then $\operatorname{AP}\left(\mathcal{A}^{\prime}\right)^{\prime}$ is amenable.

Proof. This is immediate, as $\operatorname{AP}\left(\mathcal{A}^{\prime}\right)^{\prime}$ can be identified with a quotient of a suitable ultrapower of $\mathcal{A}$.

We note that for some algebras $\mathcal{A}, \operatorname{AP}\left(\mathcal{A}^{\prime}\right)$ can be trivial. For example, let $E$ be an infinite dimensional Banach space, and let $\mathcal{A}=\mathcal{A}(E)$ be the algebra of approximable operators on $E$. Then the dual of $\mathcal{A}(E)$ is $\mathcal{I}\left(E^{\prime}\right)$, then space of integral operators on $E$. For $U \in \mathcal{I}\left(E^{\prime}\right), T \in \mathcal{A}(E)$ and $\mu \otimes x \in \mathcal{A}(E)$, we see that

$$
\langle(\mu \otimes x) \cdot U, T\rangle=\langle U, T(\mu \otimes x)\rangle=\langle U, \mu \otimes T(x)\rangle=\langle U(\mu), T(x)\rangle=\langle U(\mu) \otimes x, T\rangle .
$$

Let $U \in \mathcal{I}\left(E^{\prime}\right)$ be non-zero, and choose $\mu \in E^{\prime}$ with $\|\mu\|=1$ and such that $\lambda=U(\mu)$ is non-zero. Define a map $R_{\lambda}: E \rightarrow \mathcal{I}\left(E^{\prime}\right)$ by $R_{\lambda}(x)=\lambda \otimes x$, so that $R_{\lambda}$ is an isomorphism onto its range. We hence have that $(\mu \otimes x) \cdot U=R_{\lambda}(x)$ for each $x \in E$. As

$$
\{S \cdot U: S \in \mathcal{A}(E),\|S\| \leq 1\} \supseteq\{U(\mu) \otimes x: x \in E,\|x\| \leq 1\}=\left\{R_{\lambda}(x):\|x\| \leq 1\right\},
$$

we see if $U \in \operatorname{AP}\left(\mathcal{A}^{\prime}\right)$, then $R_{\lambda}$ is compact, which implies that $E$ is finite-dimensional, a contradiction. We conclude that $\operatorname{AP}\left(\mathcal{A}^{\prime}\right)=\{0\}$.

When $\mathcal{A}=L^{1}(G)$, however, $\operatorname{AP}\left(\mathcal{A}^{\prime}\right)$ is often large. We write $\operatorname{AP}(G)$ for $\operatorname{AP}\left(\mathcal{A}^{\prime}\right)$. For $g \in G$ define

$$
L_{g}: L^{\infty}(G) \rightarrow L^{\infty}(G) ; \quad L_{g}(\mu)(h)=\mu\left(g^{-1} h\right) \quad\left(\mu \in L^{\infty}(G), h \in G\right)
$$


When $G$ is discrete, we see that for $\mu \in L^{\infty}$, the set $\left\{a \cdot \mu: a \in \ell^{1}(G),\|a\| \leq 1\right\}$ is contained in the closure of the absolutely convex hull of the set $\left\{L_{g}(\mu): g \in G\right\}$. Hence $\mu \in \operatorname{AP}(G)$ if and only if the set of translates $\left\{L_{g}(\mu): g \in G\right\}$ is relatively compact in $L^{\infty}(G)$. By a more intricate argument, we can show that this is true for general $G$ (compare with the argument in [33]).

For more details on $\operatorname{AP}(G)$, see for example [22, Section 3.2.16] and [23, Theorem 12.4.15]. We have that $\operatorname{AP}(G)$ is a unital $\mathrm{C}^{*}$-subalgebra of $L^{\infty}(G)$, so that $\operatorname{AP}(G)=C(K)$, for some compact space $K$. We denote $K$ by $G^{\mathrm{AP}}$, so that $\operatorname{AP}\left(\mathcal{A}^{\prime}\right)^{\prime}=M\left(G^{\mathrm{AP}}\right)$, the space of measures on $G^{\mathrm{AP}}$. Each member of $G$ induces a character on $\operatorname{AP}(G)$, and this leads to a canonical map $G \rightarrow G^{\mathrm{AP}}$ which has dense range. We can use this to extend the product on $G$ to a product on $G^{\mathrm{AP}}$, which turns $G^{\mathrm{AP}}$ into a compact group. We can check that the induced product on $\operatorname{AP}\left(\mathcal{A}^{\prime}\right)^{\prime}$ agrees with the convolution product on $M\left(G^{\mathrm{AP}}\right)$. In fact, $G^{\mathrm{AP}}$ has the following universal property. If $H$ is any compact group, and $\phi: G \rightarrow H$ is a continuous homomorphism, then $\phi$ factors through to canonical map $G \rightarrow G^{\mathrm{AP}}$. In this sense, $G^{\mathrm{AP}}$ is the maximal compact group which contains a dense homomorphic image of $G$.

In general, the canonical map $G \rightarrow G^{\mathrm{AP}}$ need not be an injection. When it is, equivalently, when $\operatorname{AP}(G)$ separates the points of $G$, we say that $G$ is maximally almost periodic. Obviously all compact groups are maximally almost periodic, and abelian groups $G$ are also maximally almost periodic, which follows as the characters of $G$ separate the points of $G$. At the other extreme, if $\operatorname{AP}(G)$ is the linear span of the constant function in $L^{\infty}(G)$, then $G$ is minimally almost periodic. This is equivalent to the statement that the only continuous homomorphisms from $G$ to a compact group are trivial.

There exist amenable, minimally almost periodic groups, as the following example, due to George Willis, shows. Let $\mathrm{FSym}(\mathbb{N})$ be the collection of permutations of the natural numbers which fix all but finitely many elements. Let $G=\operatorname{Alt}(\mathbb{N})$ be the index 2 subgroup of even permutations. The Alt $(\mathbb{N})$ is a simple group (see [13, Corollary 3.3A]) and as it is the direct limit of finite groups, it is amenable. Suppose that $\operatorname{AP}(G)$ is not trivial, so that $G$ admits some non-trivial homomorphism into a compact group. By the representation theory of compact groups (essentially, the Peter-Weil theorem), it follows that there is a non-trivial homomorphism of $G$ into a matrix group $G L_{n}(\mathbb{C})$. As $G$ is simple, such a homomorphism is injective. By a theorem of Tits (see [24, Theorem 3.10]) it follows that $G$ contains a normal, solvable group of finite index. This is a contraction, and so we see that $\operatorname{AP}(G)$ is trivial.

Theorem 5.9. Let $G$ an infinite compact group, or an infinite abelian locally compact group. Then $L^{1}(G)$ is amenable, but not ultra-amenable.

Proof. It is well know that for such groups $G, L^{1}(G)$ is amenable. Suppose that $L^{1}(G)$ is ultraamenable, so by the above proposition, we see that $M\left(G^{\mathrm{AP}}\right)$ is amenable. By [5], this implies that $G^{\mathrm{AP}}$ is amenable and discrete, but as $G^{\mathrm{AP}}$ is compact, we have that $G^{\mathrm{AP}}$ is finite. However, we remarked above that $G$ is maximally periodic, and so we see that $G$ is also finite, a contradiction.

\subsection{Discrete Group algebras}

We wish to develop a little theory for discrete group algebras. This will motivate some technical and obtuse constructions below, which will work for many non-compact group. Let $G$ be a discrete group, and consider the Banach algebra $\ell^{1}(G)$. We write $\delta_{g}$ for the point mass at $g \in G$, so every $a \in \ell^{1}(G)$ can be written as $a=\sum_{g \in G} a_{g} \delta_{g}$ for some family of scalars $\left(a_{g}\right)_{g \in G}$ such that $\|a\|=\sum_{g}\left|a_{g}\right|$. Let $\mathcal{U}$ be an ultrafilter on an index set $I$, so we can form the ultrapower $\left(\ell^{1}(G)\right)_{\mathcal{U}}$. We can also form the ultrapower of $G$, denoted by $(G)_{\mathcal{U}}$. This is the set of all families $\left(g_{i}\right)_{i \in I}$ of 
elements of $G$, quotiented by the equivalence relation

$$
\left(g_{i}\right) \sim\left(h_{i}\right) \Leftrightarrow\left\{i \in I: g_{i}=h_{i}\right\} \in \mathcal{U} .
$$

Then $(G)_{\mathcal{U}}$ becomes a (discrete) group for the pointwise product, and we have a canonical map $G \rightarrow(G)_{\mathcal{U}}$ formed by sending $g \in G$ to the constant family $(g)$.

For $1 \leq p<\infty$, define a map $\psi_{p}: \ell^{p}\left((G)_{\mathcal{U}}\right) \rightarrow\left(\ell^{p}(G)\right)_{\mathcal{U}}$ by

$$
\psi_{p}\left(\delta_{g}\right)=\left(\delta_{g_{i}}\right)_{i \in I} \quad\left(g=\left(g_{i}\right) \in(G)_{\mathcal{U}}\right) .
$$

If $\left(g_{i}\right) \sim\left(h_{i}\right)$ in $(G)_{\mathcal{U}}$ then

$$
\left\{i \in I:\left\|\delta_{g_{i}}-\delta_{g_{i}}\right\|=0\right\}=\left\{i \in I: g_{i}=h_{i}\right\} \in \mathcal{U}
$$

showing that $\psi_{p}$ is well-defined. An analogous calculation shows that if we extend $\psi_{p}$ by linearity and continuity, then $\psi_{p}$ is an isometry onto its range. Let $\psi_{0}: c_{0}\left((G)_{\mathcal{U}}\right) \rightarrow\left(c_{0}(G)\right)_{\mathcal{U}}$ be the analogous map. Then it is easy to check that when $1<p<\infty$ and $p^{-1}+p^{\prime-1}=1$, then $\psi_{p^{\prime}}^{\prime} \circ \psi_{p}$ is the identity on $\ell^{p}\left((G)_{\mathcal{U}}\right)$. Similarly $\psi_{0}^{\prime} \circ \psi_{1}$ is the identity on $\ell^{1}\left((G)_{\mathcal{U}}\right)$, where as usual, we treat $\left(\ell^{1}(G)\right)_{\mathcal{U}}$ as a subspace of $\left(c_{0}(G)\right)_{\mathcal{U}}^{\prime}$. For $1 \leq p<\infty$, we can hence identify $\ell^{p}((G) \mathcal{U})$ with a 1-complemented subspace of $\left(\ell^{p}(G)\right) \mathcal{U}$. This identification respects the identification of $\ell^{p}(G)$ in either $\ell^{p}\left((G)_{\mathcal{U}}\right)$ or $\left(\ell^{p}(G)\right) \mathcal{U}$. Let $I_{p}$ be the obvious complementary subspace to $\ell^{p}((G) \mathcal{U})$ in $\left(\ell^{p}(G)\right)_{\mathcal{U}}$, that is, $I_{p}$ is the kernel of $\psi_{p^{\prime}}^{\prime}$, or $\psi_{0}^{\prime}$, as appropriate.

Lemma 5.10. We may identify $I_{p}$ with the collection of equivalence classes in $\left(\ell^{p}(G)\right) \mathcal{u}$ represented by sequences $\left(x_{i}\right)_{i \in \mathbb{N}}$ with $\lim _{i \rightarrow \mathcal{U}}\left\|x_{i}\right\|_{\infty}=0$. Furthermore, $I_{1}$ is an ideal in the algebra $\left(\ell^{1}(G)\right)_{\mathcal{U}}$.

Proof. Suppose that $\left(x_{i}\right) \in\left(\ell^{p}(G)\right)_{\mathcal{U}}$ is such that $\lim _{i \rightarrow \mathcal{U}}\left\|x_{i}\right\|_{\infty}>0$. For each $i$, let $x_{i}=\left(x_{g}^{(i)}\right)_{g \in G} \in$ $\ell^{p}(G)$. Hence there exists $\delta>0$ and $U \in \mathcal{U}$ with a function $k: U \rightarrow G$ such that $\left|x_{k(i)}^{(i)}\right| \geq \delta$ for $i \in U$. Extend $k$ to $I$ in an arbitrary way, so we see that

$$
\lim _{i \rightarrow \mathcal{U}}\left|\left\langle\delta_{k(i)}, x_{i}\right\rangle\right| \geq \delta
$$

and so $\left(x_{i}\right)$ does not annihilate $\left(\delta_{k(i)}\right) \in \ell^{p}((G) \mathcal{U})$. Hence $\left(x_{i}\right) \notin I_{p}$, as required.

Now suppose that $\left(x_{i}\right) \in\left(\ell^{p}(G)\right)_{\mathcal{U}}$ is such that $\lim _{i \rightarrow \mathcal{U}}\left\|x_{i}\right\|_{\infty}=0$. For any map $k: I \rightarrow G$, we see that

$$
\lim _{i \rightarrow \mathcal{U}}\left|\left\langle\delta_{k(i)}, x_{i}\right\rangle\right| \leq \lim _{i \rightarrow \mathcal{U}}\left\|x_{i}\right\|_{\infty}=0
$$

so that $\left(x_{i}\right)$ annihilates $\left(\delta_{k(i)}\right) \in \ell^{p}\left((G)_{\mathcal{U}}\right)$. By linearity and continuity, $\left(x_{i}\right) \in I_{p}$, as required.

Finally, consider $\left(x_{i}\right) \in I_{1}$ and let $\left(a_{i}\right) \in\left(\ell^{1}(G)\right)_{\mathcal{U}}$, so that

$$
\begin{aligned}
\lim _{i \rightarrow \mathcal{U}}\left\|a_{i} x_{i}\right\|_{\infty} & =\lim _{i \rightarrow \mathcal{U}} \sup _{g \in G}\left|\sum_{h \in G} a_{h}^{(i)} x_{h^{-1} g}^{(i)}\right| \leq \lim _{i \rightarrow \mathcal{U}} \sup _{g \in G} \sum_{h \in G}\left|a_{h}^{(i)}\right|\left|x_{h^{-1} g}^{(i)}\right| \\
& \leq \lim _{i \rightarrow \mathcal{U}}\left\|x_{i}\right\|_{\infty}\left\|a_{i}\right\|_{1}=0,
\end{aligned}
$$

so that $\left(a_{i} x_{i}\right) \in I_{1}$. Hence $I_{1}$ is a left-ideal, and similarly $I_{1}$ is a right-ideal.

Theorem 5.11. Let $G$ be an infinite discrete group, and let $\mathcal{U}$ be a countably incomplete ultrafilter. Then $\left(\ell^{1}(G)\right)_{\mathcal{U}}$ is not amenable. In particular, $\ell^{1}(G)$ is not ultra-amenable.

Proof. As $\mathcal{U}$ is countably incomplete, we may suppose that $\mathcal{U}$ is an ultrafilter on $\mathbb{N}$ (compare with the proofs of Theorem 6.3 or Proposition 7.1 in [16]). Suppose that $\left(\ell^{1}(G)\right)_{\mathcal{U}}$ is amenable. Then $I_{1}$ is a complemented ideal in an amenable Banach algebra, and so $I_{1}$ is amenable (see [30, 
Theorem 2.3.7]). In particular, $I_{1}$ has a bounded approximate identity. We shall show that this leads to contradiction, as required.

Let $H$ be a countably infinite subgroup of $G$, and choose a sequence $\left(g_{n}\right)$ in $H$ as follows. Let $g_{1}$ be arbitrary. Suppose we have chosen $g_{1}, \cdots, g_{n}$. Consider the set

$$
B_{n}=\left(g_{i}\right)_{i=1}^{n} \cup\left(g_{i} g_{j}^{-1} g_{k}\right)_{1 \leq i, j, k \leq n}
$$

which is finite. We simply choose $g_{n+1} \in H \backslash B_{n}$.

Let $e_{G}$ be the unit of $G$, and let $g \in H$ with $g \neq e_{G}$. Suppose that $g_{i} g=g_{j}$ for some $i, j \geq 1$. Let $t \geq 1$ be minimal such that, for some $m \geq 1$, we have that $g=g_{t}^{-1} g_{m}$ or $g=g_{m}^{-1} g_{t}$. By minimality, $t<m$, and so $g_{i} g \in B_{m}$ for $1 \leq i \leq m$. Thus $g_{n} \neq g_{i} g$, equivalently $g \neq g_{i}^{-1} g_{n}$, for any $n>m$ and $i \leq m$. Similarly, $g_{i} g^{-1} \in B_{m}$ for $i \leq m$, and so $g \neq g_{n}^{-1} g_{i}$ for $n>m$ and $i \leq m$.

Suppose that $g=g_{t}^{-1} g_{m}$, and let $r, s \geq 1$ be such that $g=g_{r}^{-1} g_{s}$ and $(r, s) \neq(t, m)$. If $r=t$ then $g=g_{t}^{-1} g_{m}=g_{t}^{-1} g_{s}$ so that $g_{m}=g_{s}$, that is, $m=s$, a contradiction. Similarly $m \neq s$. By minimality, $r, s \geq t$, so actually $r>t$. By the above, $r, s \leq m$, so actually $s<m$. If $r<m$ then $g_{t} g \in B_{m-1}$ and so $g_{m} \neq g_{t} g$, a contradiction. Hence $r=m$ and so $g=g_{t}^{-1} g_{m}=g_{m}^{-1} g_{s}$, so that $g_{s}=g_{m} g_{t}^{-1} g_{m}$, and hence $s$ must be unique. A similar argument works when $g=g_{m}^{-1} g_{t}$. We conclude that

$$
\mid\left\{k \geq 1: g_{k} g=g_{m} \text { for some } m \geq 1\right\} \mid \leq 2 \quad\left(g \in H, g \neq e_{G}\right)
$$

Set $x=\chi_{\left\{g_{n}: n \in \mathbb{N}\right\}} \in \ell^{\infty}(G)$, so that $\|x\|=1$. For each $n \in \mathbb{N}$, let

$$
a_{n}=\frac{1}{n} \sum_{i=1}^{n} \delta_{g_{i}} \in \ell^{1}(G)
$$

so that $\left\|a_{n}\right\|_{1}=1$. We have that

$$
\left\langle x \cdot a_{n}, \delta_{e_{G}}\right\rangle=\frac{1}{n} \sum_{i=1}^{n}\left\langle x, \delta_{g_{i}}\right\rangle=1 .
$$

Now let $g \in H$ with $g \neq e_{G}$. Then, by the above,

$$
\left|\left\langle x \cdot a_{n}, \delta_{g}\right\rangle\right|=\frac{1}{n} \sum_{i=1}^{n}\left\langle x, \delta_{g_{i} g}\right\rangle=\frac{1}{n} \mid\left\{1 \leq i \leq n: g_{i} g=g_{k} \text { for some } k \geq 1\right\} \mid \leq \frac{2}{n} .
$$

If $g \in G \backslash H$, then clearly $\left\langle x, \delta_{g_{i} g}\right\rangle=0$, as $g_{i} g \notin H$, for any $i \geq 1$. Let $a=\left(a_{n}\right) \in\left(\ell^{1}(G)\right) \mathcal{U}$, so clearly $a \in I_{1}$, and we see that $x \cdot a=e_{e_{G}}$ in $\left(\ell^{\infty}(G)\right) \mathcal{U}$. Here $e_{e_{G}}$ refers to the point mass at $e_{G}$, namely the same function as $\delta_{e_{G}}$, but now treated as a member of $c_{0}(G) \subseteq \ell^{\infty}(G)$.

By assumption, $I_{1}$ has a bounded approximate identity, so in particular, there exists $b=\left(b_{n}\right) \in$ $I_{1}$ with $\|a-a b\|<1 / 2$. Hence $\left|\left\langle x \cdot a, \delta_{e_{G}}-b\right\rangle\right|=|\langle x, a-a b\rangle|<1 / 2$, as $\|x\|_{\infty}=1$. However, from the above, $\left\langle x \cdot a, \delta_{e_{G}}-b\right\rangle=\left\langle e_{e_{G}}, \delta_{e_{G}}-b\right\rangle=1-\lim _{i \rightarrow \mathcal{U}}\left\langle e_{e_{G}}, b_{i}\right\rangle=1$, as $b \in I_{1}$. This contradiction completes the proof.

\subsection{General groups}

We start by making some observations about quotients of groups.

Proposition 5.12. Let $G$ be a locally compact group such that $L^{1}(G)$ is ultra-amenable, and let $H$ be a closed normal subgroup of $G$. If $G / H$ is compact, abelian or discrete, then $G / H$ is finite. 
Proof. As detailed in [22, Section 1.9.12], we have a surjective algebra homomorphism $L^{1}(G) \rightarrow$ $L^{1}(G / H)$. By Proposition [5.2, we see that $L^{1}(G / H)$ is ultra-amenable. The result now follows from Theorem 5.9 and Theorem 5.11 .

In particular, by considering the modular function of the Haar measure on $G$ (see [22, Section 1.9] or [3, Section 3.3]), we see that if $L^{1}(G)$ is ultra-amenable, then $G$ is unimodular, as otherwise, a quotient of $G$ would be isomorphic to an infinite subgroup of $\left(\mathbb{R}_{>0}, \times\right)$, which is abelian. Similarly, if $L^{1}(G)$ is ultra-amenable, then the derived subgroup $G^{\prime}$ of $G$ (see [23, Section 12.1]) must be "large", in the sense that $G / G^{\prime}$ is finite.

We now wish to generalise the arguments used above for discrete groups. Let $G$ be a locally compact group, and let $\mathcal{U}$ be an ultrafilter on an index set $I$. Let $I_{1} \subseteq\left(L^{1}(G)\right)_{\mathcal{U}}$ be the collection of elements $x \in\left(L^{1}(G)\right)_{\mathcal{U}}$ such that $x$ has a representation of the form $\left(x_{i}\right)_{i \in I}$ where $x_{i} \in C_{0}(G)$ for each $i$, and $\lim _{i \rightarrow \mathcal{U}}\left\|x_{i}\right\|_{\infty}=0$. It is easy to verify that $I_{1}$ is a subspace of $\left(L^{1}(G)\right)_{\mathcal{U}}$, and as $C_{0}(G) \cap L^{1}(G)$ is dense in $L^{1}(G)$, it follows that $I_{1}$ is closed.

Lemma 5.13. With notation as above, $I_{1}$ is an ideal in $\left(L^{1}(G)\right)_{\mathcal{U}}$.

Proof. Let $x \in I_{1}$, so $x=\left(x_{i}\right)$ with $x_{i}$ as above. Let $a=\left(a_{i}\right) \in\left(L^{1}(G)\right)_{\mathcal{U}}$, where by density, we may suppose that $a_{i} \in C_{0}(G)$ for each $i$. Then $a_{i} x_{i} \in C_{0}(G)$ for each $i$, and we have that $\left\|a_{i} x_{i}\right\|_{\infty} \leq\left\|a_{i}\right\|_{\infty}\left\|x_{i}\right\|_{1}$, from which it follows that $a x=\left(a_{i} x_{i}\right) \in I_{1}$, so we see that $I_{1}$ is a left-ideal. Similarly $I_{1}$ is a right-ideal.

We cannot, in general, show that $I_{1}$ is complemented. However, we shall show that $I_{1}$ is weaklycomplemented. That is,

$$
I_{1}^{\perp}=\left\{\mu \in\left(L^{1}(G)\right)_{\mathcal{U}}^{\prime}:\langle\mu, x\rangle=0\left(x \in I_{1}\right)\right\}
$$

is complemented in $\left(L^{1}(G)\right)_{\mathcal{U}}^{\prime}$.

Proposition 5.14. With notation as above, $I_{1}$ is weakly-complemented.

Proof. We shall sketch this, as the details are very similar to ideas used to deal with dual Banach algebras in Section 3.1 above. Let $\phi$ be the composition of the isometric inclusions

$$
\left(L^{1}(G)\right)_{\mathcal{U}} \rightarrow(M(G))_{\mathcal{U}}=\left(C_{0}(G)^{\prime}\right)_{\mathcal{U}} \rightarrow\left(C_{0}(G)\right)_{\mathcal{U}}^{\prime}
$$

so that $\phi^{\prime}:\left(C_{0}(G)\right)_{\mathcal{U}}^{\prime \prime} \rightarrow\left(L^{1}(G)\right)_{\mathcal{U}}^{\prime}$ is a quotient map (or metric surjection). As $\left(C_{0}(G)\right)_{\mathcal{U}}$ is a $\mathrm{C}^{*}$-algebra, we see that $\left(C_{0}(G)\right)_{\mathcal{U}}^{\prime \prime}$ is a von Neumann algebra. We can verify that the kernel of $\phi^{\prime}$ is an ideal in $\left(C_{0}(G)\right)_{\mathcal{U}}^{\prime \prime}$, and so $\left(L^{1}(G)\right)_{\mathcal{U}}^{\prime}$ becomes a $\mathrm{C}^{*}$-algebra. As $\left(L^{1}(G)\right)_{\mathcal{U}}^{\prime}$ is a dual space, we see that $\left(L^{1}(G)\right)_{\mathcal{U}}^{\prime}$ is a commutative von Neumann algebra. Notice that $\left(L^{1}(G)\right)_{\mathcal{U}}^{\prime}$ isometrically contains $\left(L^{\infty}(G)\right)_{\mathcal{U}}$, and it is not hard to show that $\left(L^{\infty}(G)\right)_{\mathcal{U}}$ becomes a *-subalgebra of $\left(L^{1}(G)\right)_{\mathcal{U}}^{\prime}$.

We can check that $I_{1}^{\perp}$ is a $*$-subalgebra of $\left(L^{1}(G)\right)_{\mathcal{U}}^{\prime}$. As $I_{1}^{\perp}$ is weak ${ }^{*}$-closed, we see that $I_{1}^{\perp}$ is a commutative von Neumann algebra, and is hence injective (see [30, Section 6.2]). Hence there is a (contractive) projection $\left(L^{1}(G)\right)_{\mathcal{U}}^{\prime} \rightarrow I_{1}^{\perp}$, as required.

We now make a temporary definition. Let $G$ be a non-compact, locally compact group. We shall say that $G$ is relatively-[IN] if there exists a compact, symmetric, non-null (with respect to Haar measure) set $K$ in $G$ and a subset $A \subseteq G$ whose closure is not compact, such that $a K=K a$ for each $a \in A$. We shall say that $(K, A)$ is a witness. We recall that if we can take $A=G$, then $G$ is an [IN]-group (see [23, Section 12.1.8] for further details of this class of groups).

Lemma 5.15. Let $G$ be a relatively-[IN] group witnessed by $(K, A)$. Let $A_{0}$ be the closed subgroup generated by $A$. Then $a K=K a$ for $a \in A_{0}$. Let $K_{0}$ be the closed subgroup generated by $K$. Then also a $K_{0}=K_{0}$ a for $a \in A_{0}$. Finally, if $G_{0}$ is the closed subgroup of $G$ generated by $K_{0}$ and $A_{0}$, then $g K_{0}=K_{0} g$ for $g \in G_{0}$. 
Proof. As $K$ is symmetric, we see that if $g, h \in A$, then $g^{-1} K=K g^{-1}$, and $g h K=g K h=K g h$. Hence $K$ is invariant under the action of the subgroup generated by $A$. Let $\left(a_{\alpha}\right)$ be a net in the subgroup generated by $A$ converging to $a \in G$. For $k \in K$, the net $\left(a_{\alpha} k a_{\alpha}^{-1}\right)$ is in $K$ and converges to $a k a^{-1}$, so as $K$ is closed, $a k a^{-1} \in K$, and so we see that $a K=K a$. Thus $K$ is invariant under the action of $A_{0}$.

As $K$ is symmetric, the subgroup generated by $K$ is simply $\bigcup_{n>1} K^{n}$, and it is clear that this is invariant under the action of $A_{0}$. Let $a \in A_{0}$ and let $\left(k_{\alpha}\right)$ be a net in the subgroup generated by $K$ tending to $k \in G$. Then $a k_{\alpha} a^{-1} \rightarrow a k a^{-1}$, showing that $a k a^{-1} \in K_{0}$. Hence $K_{0}$ is invariant under the action of $A_{0}$.

Let $G_{1}$ be the subgroup of $G$ generated by $A_{0}$ and $K_{0}$. As $K_{0}$ is invariant under $A_{0}$, we see that $G_{1}=A_{0} K_{0}=K_{0} A_{0}$, and so clearly $K_{0}$ is invariant under the action of $G_{1}$. Again, a continuity argument shows that the same holds for $G_{0}$.

We shall see later that we really only care about subgroups, and so the above shows that being a relatively-[IN] group is rather similar to having a closed subgroup which is [IN]. However, we cannot in general show that $K$ (as opposed to $K_{0}$ ) is invariant for $G_{0}$. Indeed, $K$ is $G_{0}$-invariant if and only if $K$ is $K$-invariant $(k K=K k$ for each $k \in K)$.

However, we can always assume that for a witness $(K, A)$, we have that $A$ is a closed noncompact subgroup of $G$.

Theorem 5.16. Let $G$ be a non-compact group which is relatively-[IN]. Let $\mathcal{U}$ be a countably incomplete ultrafilter. Then $\left(L^{1}(G)\right)_{\mathcal{U}}$ is not amenable. In particular, $L^{1}(G)$ is not ultra-amenable.

Proof. As in the proof of Theorem 5.11 above, we may suppose that $\mathcal{U}$ is an ultrafilter on the index set $\mathbb{N}$. If $\left(L^{1}(G)\right)_{\mathcal{U}}$ is amenable, then as $I_{1}$ is a weakly-complemented ideal, then $I_{1}$ is amenable as well, by [30, Theorem 2.3.7]. Again, in particular, $I_{1}$ has a bounded approximate identity. Let $(K, A)$ be a witness to the fact that $G$ is relatively-[IN].

We choose a sequence $\left(g_{n}\right)$ in $A$ as follows. Let $g_{1} \in A$ be arbitrary. Suppose we have chosen $g_{1}, \cdots, g_{n}$, and let

$$
B_{n}=\bigcup_{i=1}^{n} g_{i} K K \cup \bigcup_{i=1}^{n} g_{i} K^{4} \cup \bigcup_{i=1}^{n} g_{i} K^{8} \cup \bigcup_{i, j, k=1}^{n} g_{i} g_{j}^{-1} g_{k} K^{4}
$$

so that $B_{n}$ is compact in $G$. We can hence choose $g_{n+1} \notin B_{n}$, as $A$ is not compact. Then, for $k \leq n$, we see that $g_{n+1} \notin g_{k} K K=K K g_{k}$, so that $g_{n+1} g_{k}^{-1} \notin K K$. Similarly, as $(K K)^{-1}=K K$, we see that $g_{k} g_{n+1}^{-1} \notin K K$ for $k \leq n$.

Let $g \notin K K$, and let $t \geq 1$ be minimal such that, for some $m \geq 1$, we have that $g \in g_{m} g_{t}^{-1} K K$ or $g \in g_{t} g_{m}^{-1} K K$. As $g \notin K K$, by minimality, we have that $t<m$. Let $r, s \geq 1$ with $g \in g_{r} g_{s}^{-1} K K$, so by minimality, $r, s \geq t$, so actually, $r>t$ or $s>t$.

Suppose that $r, s<m$, so that either $g \in g_{m} g_{t}^{-1} K K$, so that $g_{m} \in g K K g_{t} \subseteq g_{r} g_{s}^{-1} K^{4} g_{t}=$ $g_{r} g_{s}^{-1} g_{t} K^{4} \subseteq B_{m-1}$, a contradiction; or $g \in g_{t} g_{m}^{-1} K K=K K g_{t} g_{m}^{-1}$, so that $g_{m} \in g^{-1} K K g_{t} \subseteq$ $g_{s} g_{r}^{-1} K^{4} g_{t}=g_{s} g_{r}^{-1} g_{t} K^{4} \subseteq B_{m-1}$, a contradiction. Hence $r \geq m$ or $s \geq m$.

As $r \neq s$, either $r<s$ or $s<r$. If $r<s$, then the argument in the previous paragraph shows that we do not have that $m, t<s$, that is, $m \geq s$. Similarly, if $s<r$ then $m \geq r$. We conclude that $m \geq \max (r, s) \geq m$, so that $m=\max (r, s)$.

Suppose that $g \in g_{m} g_{t}^{-1} K K$. If $m=r>s$, then $g_{m}^{-1} g \in g_{t}^{-1} K K \cap g_{s}^{-1} K K$. If $t>s$ then $g_{t} \in K^{4} g_{s}$, a contradiction, so by symmetry, $t=s$. Otherwise $m=s>r$, in which case $g \in g_{m} g_{t}^{-1} K K \cap g_{r} g_{m}^{-1} K K$ so that $g_{m} g_{t}^{-1} g_{m} \in g_{r} K^{4}$. Suppose that there exists $r^{\prime}$ with $r^{\prime}<s$ and $g \in g_{r^{\prime}} g_{m}^{-1} K K$, so that $g_{m} g_{t}^{-1} g_{m} \in g_{r^{\prime}} K^{4}$. Suppose that $r>r^{\prime}$, so that $g_{r} \in g_{r^{\prime}} K^{8}$, a contradiction, so by symmetry, we conclude that $r=r^{\prime}$. Hence, $r$ is unique. 
An analogous argument works when $g \in g_{t} g_{m}^{-1} K K$, showing that in all cases,

$$
\mid\left\{1 \leq l \leq n: g \in g_{k} g_{l}^{-1} K K \text { for some } k \geq 1\right\} \mid \leq 2 \quad(n \geq 1, g \in G \backslash K K) .
$$

For a measurable subset $B \subseteq G$ we let $\chi_{B}$ be the indicator function of $B$, so that $\chi_{B} \in L^{\infty}(G)$. When $B$ has finite measure, we have that $\chi_{B} \in L^{1}(G)$; write $\chi_{B}^{1}$ in this case. Let $x \in L^{\infty}(G)$ be defined by the following formal sum

$$
x=\sum_{n=1}^{\infty} \chi_{g_{n} K}
$$

which makes sense, as by construction, $g_{n} K \cap g_{m} K=\emptyset$ when $n \neq m$. We see that $\|x\|_{\infty}=1$. For each $n \geq 1$, let

$$
a_{n}=\frac{1}{n|K|} \sum_{k=1}^{n} \chi_{g_{k} K}^{1} \in L^{1}(G)
$$

so that $\left\|a_{n}\right\|=1$, and hence $a=\left(a_{n}\right) \in\left(L^{1}(G)\right)_{\mathcal{U}}$. Notice that $\left\langle x, a_{n}\right\rangle=1$ for all $n$. Define $f \in L^{\infty}(G)$ by $f=|K|^{-1} \chi_{K}^{1} \cdot \chi_{K}$, so that

$$
f(g)=\frac{1}{|K|} \int_{G} \chi_{K}(g h) \chi_{K}^{1}(h) d h=\frac{1}{|K|} \int_{K} \chi_{g^{-1} K}(h) d h=\frac{\left|K \cap g^{-1} K\right|}{|K|} \quad(g \in G) .
$$

Then $f$ is continuous, $\|f\|_{\infty} \leq 1$ and $f\left(e_{G}\right)=1$. Furthermore, $f(g) \neq 0$ only when $g \in K K$. For $s, t \in G$, we see that

$$
\begin{aligned}
|K|^{-1}\left(\chi_{s K}^{1} \cdot \chi_{t K}\right)(g) & =\frac{1}{|K|} \int_{G} \chi_{t K}(g h) \chi_{s K}^{1}(h) d h=\frac{1}{|K|} \int_{G} \chi_{K}\left(t^{-1} g h\right) \chi_{K}^{1}\left(s^{-1} h\right) d h \\
& =\frac{1}{|K|} \int_{G} \chi_{K}\left(t^{-1} g s h\right) \chi_{K}^{1}(h) d h=f\left(t^{-1} g s\right) .
\end{aligned}
$$

Hence $\left(\chi_{s K}^{1} \cdot \chi_{t K}\right)(g) \neq 0$ only when $g \in t K K s^{-1}=t s^{-1} K K$, that is, $s t^{-1} g \in K K$.

For $g \in G$, we have that

$$
\left(a_{n} \cdot x\right)(g)=\frac{1}{n} \sum_{k=1}^{\infty} \sum_{l=1}^{n}|K|^{-1}\left(\chi_{g_{l} K}^{1} \cdot \chi_{g_{k} K}\right)(g)=\frac{1}{n} \sum_{k=1}^{\infty} \sum_{l=1}^{n} f\left(g_{k}^{-1} g g_{l}\right) .
$$

Hence, for $g \notin K K$, we see that

$$
\left|\left(a_{n} \cdot x\right)(g)\right| \leq \frac{1}{n} \mid\left\{1 \leq l \leq n: g_{l} g_{k}^{-1} g \in K K \text { for some } k \geq 1\right\} \mid \leq \frac{2}{n},
$$

from the above. If $b=\left(b_{n}\right) \in I_{1}$ then $\left\|b_{n}\right\|_{\infty} \rightarrow 0$, and so $\left\|\left.b_{n}\right|_{K K}\right\|_{1} \rightarrow 0$, and from this it follows that $b a \neq a$, exactly as in the proof of Theorem 5.11. This contradiction completes the proof.

Notice that, for abelian groups $G$, the above improves upon Theorem [5.9, as Theorem 5.9 only tells us that $\left(L^{1}(G)\right)_{\mathcal{U}}$ is not amenable when there is a surjection $\left(L^{1}(G)\right)_{\mathcal{U}} \rightarrow A P(G)^{\prime}$; unless $G$ is sufficiently "small", we cannot necessarily take $\mathcal{U}$ to be an ultrafilter on a countable set.

We conclude with the following.

Theorem 5.17. Let $G$ be a locally compact group such that $L^{1}(G)$ is ultra-amenable. Then $G$ is finite; or $G$ satisfies the following:

1. $G$ is amenable;

2. $G$ is not compact nor a relative-[IN] group (so that $G$ is not abelian or discrete); 


\section{3. $A P(G)$ is finite-dimensional;}

4. if $H$ is a closed normal subgroup of $G$ then either $G / H$ is finite, or $G / H$ satisfies the above properties.

We currently do not know of any group which satisfies the above conditions, so we strongly suspect that $L^{1}(G)$ is only ultra-amenable when $G$ is finite.

We suspect that a careful argument using the ideas of Proposition 5.12 could reduce this problem to the study of totally disconnected groups (see [23, Section 12.3]), as connected groups are fairly well understood (they are pro-Lie groups, see [23, Section 12.2]). Of course, totally disconnected groups are not terribly well understood. We strongly suspect that the correct course of attack is to be improve the proof of Theorem [5.16] so that it will hold for all non-compact groups.

\section{Acknowledgments}

The results of Section 4 are mostly from the author's PhD thesis [9] completed at the University of Leeds under the financial support of the EPSRC, and the guidance of his PhD supervisors Garth Dales and Charles Read. The author would also like to thank George Willis, who proved to be an excellent source for all questions group related, and Volker Runde, for useful conversations, especially about $\mathrm{C}^{*}$-algebras. The author made great use of the unpublished notes "Ultra-methods" by Mark Smith. Finally, the author wishes to thank the anonymous referee for careful proofreading.

\section{References}

[1] C. C. Chang, H. J. Keisler, Model theory, (North-Holland Publishing Co., Amsterdam-London).

[2] M. Cowling, G. Fendler, 'On representations in Banach spaces', Math. Ann. 266 (1984) 307-315.

[3] H. G. DALEs, Banach algebras and automatic continuity, (Clarendon Press, Oxford, 2000).

[4] H. G. Dales, A. T.-M. Lau, 'The second duals of Beurling algebras', Mem. Amer. Math. Soc. 177 (2005) $191 \mathrm{pp}$.

[5] H. G. Dales, F. Ghahramani, A. Ya. Helemskit, 'The amenability of measure algebras', J. London Math. Soc. 66 (2002) 213-226.

[6] D. Dacunha-Castelle, J. L. Krivine, 'Applications des ultraproduits à l'étude des espaces et des algèbres de Banach', Studia Math. 41 (1972) 315-334.

[7] M. DAws, 'Ultrapowers of Banach algebras and modules', preprint. See arXiv:0708.4029 [math.FA]

[8] M. Daws, 'Dual Banach algebras: representations and injectivity', Studia Math. 178 (2007) 231-275.

[9] M. DAws, Banach algebras of operators, PhD. thesis, University of Leeds, 2005.

[10] M. Daws, 'Arens regularity of the algebra of operators on a Banach space', Bull. London Math. Soc. 36 (2004) 493-503.

[11] A. Defant, K. Floret, Tensor norms and operator ideals, (North-Holland Publishing Co., Amsterdam, 1993).

[12] J. Diestel, J.J. Uhl, JR., Vector measures, (American Mathematical Society, Providence, 1977).

[13] J. D. Dixon, B. Mortimer, Permutation groups, (Graduate Texts in Mathematics, 163. Springer-Verlag, New York, 1996).

[14] L. Ge, D. Hadwin, 'Ultraproducts of $C^{*}$-algebras', in Recent advances in operator theory and related topics, 305-326, Oper. Theory Adv. Appl., 127, Birkhuser, Basel, 2001. 
[15] G. Godefroy, B. Iochum, 'Arens-regularity of Banach algebras and the geometry of Banach spaces', $J$. Funct. Anal. 80 (1988) 47-59.

[16] S. HeInRICH, 'Ultraproducts in Banach space theory', J. reine angew. Math. 313 (1980) 72-104.

[17] B. Iochum, G. Loupias, 'Arens regularity and local reflexivity principle for Banach algebras', Math. Ann. 284 (1989) 23-40.

[18] R. C. James, 'Super-reflexive Banach spaces', Canad. J. Math. 24 (1972) 896-904.

[19] S. Kaijser, 'On Banach modules. I.', Math. Proc. Cambridge Philos. Soc. 90 (1981) 423-444.

[20] A. T.-M. LaU, R. J. LoY, G. A. Willis, 'Amenability of Banach and $C^{*}$-algebras on locally compact groups', Studia Math. 119 (1996) 161-178.

[21] T. W. Palmer, 'The bidual of the compact operators', Trans. Amer. Math. Soc. 288 (1985) 827-839.

[22] T. W. Palmer, Banach algebras and the general theory of ${ }^{*}$-algebras. Vol. 1., (Cambridge University Press, Cambridge, 1994).

[23] T. W. Palmer, Banach algebras and the general theory of*-algebras. Vol. 2., (Cambridge University Press, Cambridge, 2001).

[24] A. L. T. Paterson, Amenability, (Mathematical Surveys and Monographs, 29. American Mathematical Society, Providence, RI, 1988).

[25] V. I. Paulsen, R. R. Smith, 'Diagonals in tensor products of operator algebras', Proc. Edinb. Math. Soc. 45 (2002) 647-652.

[26] A. Pietsch, Operator Ideals, (North-Holland, Amsterdam, 1980).

[27] J. S. PYM, 'The convolution of functionals on spaces of bounded functions', Proc. London Math. Soc. 15 (1965) 84-104.

[28] Y. Raynaud, 'On ultrapowers of non commutative $L_{p}$ spaces', J. Operator Theory 48 (2002) 41-68.

[29] V. Runde, 'Cohen-Host type idempotent theorems for representations on Banach spaces and applications to Figà-Talamanca-Herz algebras', J. Math. Anal. Appl. 329 (2007) 736-751.

[30] V. Runde, Lectures on amenability, (Springer-Verlag, Berlin, 2002).

[31] V. Runde, 'Amenability for dual Banach algebras', Studia Math. 148 (2001) 47-66.

[32] R. RYAN, Introduction to Tensor Products of Banach Spaces, (Springer-Verlag, London, 2002).

[33] A. ÜLGER, 'Continuity of weakly almost periodic functionals on $L^{1}(G)$ ', Quart. J. Math. Oxford Ser. (2) 37 (1986) 495-497.

[34] N. J. Young, 'Periodicity of functionals and representations of normed algebras on reflexive spaces', Proc. Edinburgh Math. Soc. 20 (1976/77) 99-120.

Author's Address: St. John's College, Oxford, OX1 3JP.

Email: matt.daws@cantab.net 


\title{
A Errata
}

\begin{abstract}
Some of the results of Section 5 of this paper are incorrect; in particular, the characterisation of when an algebra is ultra-amenable, in terms of a diagonal like construction, is not proved; and Theorem 5.7 is stated wrongly. The rest of the paper is unaffected. We shall show in this erratum that Theorem 5.7 can be corrected, and that the other results of Section 5 are true if the algebra in question has a certain approximation property. 2000 Mathematics subject classification: 46B08; $46 \mathrm{~B} 28$.
\end{abstract}

Some of the results of Section 5 of [3] are incorrect. The claim (ii) $\Rightarrow$ (i) of Proposition 5.4 implicitly assumes that $\psi_{0}$ is bounded below, but this is unproven. Hence also the claim, in Corollary 5.5, that if a Banach algebra $\mathcal{A}$ is contractible then it is ultra-amenable, is unproven. Similarly (ii) $\Rightarrow(\mathrm{i})$ of Theorem 5.6 requires $\psi_{0}$ to be bounded below. The rest of the paper is unaffected. We used some of these ideas in [4, Section 4], and so this is also incorrect; an erratum has been submitted.

Firstly, we deal with correcting Theorem 5.7. We say that a $\mathrm{C}^{*}$-algebra $\mathcal{A}$ is subhomogeneous if there exists $n \in \mathbb{N}$ such that every irreducible representation of $\mathcal{A}$ has dimension at most $n$. Subhomogeneous von Neumann algebras have the special form claimed in Theorem 5.7, but this is not true for $\mathrm{C}^{*}$-algebras, see [1, Section IV.1.4] for examples. This circle of ideas was considered in [7, Theorem 2.5] but we have been unable to follow some of the proofs (in particular, the claim that $(\mathrm{A} 4) \Rightarrow(\mathrm{R} 5))$ so we provide details here.

Theorem A.1. Let $\mathcal{A}$ be a $C^{*}$-algebra. Then the following are equivalent:

1. $\mathcal{A}$ is ultra-amenable;

2. $\mathcal{A}^{\prime \prime}$ is amenable;

3. $\ell^{\infty}(\mathcal{A}, I)$ is amenable for any index set $I$;

4. $\mathcal{A}$ is subhomogeneous.

Proof. The original argument using the approximation property in [3] is correct and shows (11) $\Rightarrow(2)$. Similarly, as argued in [3] (see also [7]) if (2) holds, then $\mathcal{A}^{\prime \prime}$ has the form

$$
\mathcal{A}^{\prime \prime}=\sum_{k=1}^{n} L^{\infty}\left(X_{k}\right) \otimes \mathbb{M}_{n_{k}}
$$

where for each $k, X_{k}$ is a measure space, and $n_{k} \in \mathbb{N}$. Notice that if $\mathcal{A}^{\prime \prime}$ is of this form, then following [7], it is elementary to see that so is $l^{\infty}\left(\mathcal{A}^{\prime \prime}, I\right)$ for any index set $I$. This does imply that $\mathcal{A}$ is subhomogeneous (see [1, Proposition IV.1.4.6]) but not that $\mathcal{A}$ has the form originally claimed in [3, Theorem 5.7].

However, there is an algebraic characterisation of when $C^{*}$-algebras are subhomogeneous, see [5. Section 3.6] or [1, Section IV.1.4.5]. The algebra $\mathbb{M}_{n}$ is of dimension $n^{2}$ and so for any $r>n^{2}$, we have

$$
\sum_{\sigma \in S_{r}} \epsilon_{\sigma} x_{\sigma(1)} \cdots x_{\sigma(r)}=0
$$

for any $x_{1}, \cdots, x_{r} \in \mathbb{M}_{n}$ (this is readily seen by taking a basis). Here $S_{r}$ is the symmetric group and $\epsilon: S_{r} \rightarrow\{ \pm 1\}$ the signature. Let $r(n)$ be the smallest $r$ for which this holds for $\mathbb{M}_{n}$. Then [5. Lemma 3.6.2] shows that $r(n) \geq r(n-1)+2$ (see [1, Section IV.1.4.5] and references therein for better estimates). As irreducible representations separate the points of a $\mathrm{C}^{*}$-algebra $\mathcal{A}$, we conclude that the following are equivalent:

i. any irreducible representation of $\mathcal{A}$ is of dimension at most $n$; 
ii. for any $x_{1}, \cdots, x_{r(n)} \in \mathcal{A}$, identity (1) holds for $r=r(n)$.

Indeed, the only unclear issue is if $\pi: \mathcal{A} \rightarrow \mathcal{B}(H)$ is irreducible, with $H$ infinite dimensional, why cannot (ii) hold? However, then $\pi(\mathcal{A})$ is strongly dense in $\mathcal{B}(H)$, and $\mathbb{M}_{n+1}$ is a subalgebra of $\mathcal{B}(H)$, which is enough to show that (ii) fails.

It is clear that the second condition passes to subalgebras, and with a little thought, it is seen to pass to ultrapowers as well. Thus, if (2) holds, then $\ell^{\infty}(\mathcal{A}, I)$ is subhomogeneous and $\mathcal{A}$ is subhomogeneous, showing (44). It is reasonably easy to show that $\ell^{\infty}(\mathcal{A}, I)$ is thus nuclear (see [2, Proposition 2.7.7]), or follow [7, Theorem 2.5] for a direct argument that $\ell^{\infty}(\mathcal{A}, I)$ is thus amenable. As amenability passes to quotients, (3) $\Rightarrow(\mathbb{1})$ is clear. Finally, if (4) holds then any ultrapower of $\mathcal{A}$ is subhomogeneous and hence amenable, showing (1).

We erroneously claimed in [4] that (11) and (3) are equivalent for any Banach algebra $\mathcal{A}$. It would be interesting to know if this is true.

We shall now improve [3, Proposition 4.7], and show that the map $\psi_{0}$ is indeed bounded below for a wide class of Banach algebras $\mathcal{A}$. We leave open whether this holds for all $\mathcal{A}$ (which seems unlikely). It seems possible that similar, but stronger, conditions could characterise when $\mathcal{A}$ is ultra-amenable, but we shall not pursue this here.

Let $E$ and $F$ be Banach spaces, and let $\mathcal{U}$ be an ultrafilter on an index set $I$. As in [3], we shall suppose that $\mathcal{U}$ is countably incomplete. Recall from [3, Section 4] the map $\psi_{0}:(E)_{\mathcal{U}} \widehat{\otimes}(F)_{\mathcal{U}} \rightarrow$ $(E \widehat{\otimes} F)_{\mathcal{U}}$, defined on elementary tensors by

$$
\psi_{0}\left(\left(x_{i}\right) \otimes\left(y_{i}\right)\right)=\left(x_{i} \otimes y_{i}\right) \quad\left(\left(x_{i}\right) \in(E)_{\mathcal{U}},\left(y_{i}\right) \in(F)_{\mathcal{U}}\right) .
$$

For the following, we recall that [6, Theorem 9.1] characterises, in terms of local properties, when an ultrapower has the (bounded) approximation property.

Theorem A.2. If $(E)_{\mathcal{U}}$ has the approximation property, then $\psi_{0}$ is an injection for any $F$.

Proof. Let $\tau \in(E)_{\mathcal{U}} \widehat{\otimes}(F)_{\mathcal{U}}$ have representation $\tau=\sum_{n=1}^{\infty} x_{n} \otimes y_{n}$ with $\sum_{n}\left\|x_{n}\right\|\left\|y_{n}\right\|<\infty$. If $(E)_{\mathcal{U}}$ has the approximation property then, by [9, Proposition 4.6], if $\tau \in(E)_{\mathcal{U}} \widehat{\otimes}(F)_{\mathcal{U}}$ is non-zero, then there exist $\mu \in(E)_{\mathcal{U}}^{\prime}$ and $\lambda \in(F)_{\mathcal{U}}^{\prime}$ with

$$
0 \neq\langle\mu \otimes \lambda, \tau\rangle=\sum_{n=1}^{\infty}\left\langle\mu, x_{n}\right\rangle\left\langle\lambda, y_{n}\right\rangle .
$$

As we only care about the value of $\mu$ on the countable set $\left\{x_{n}\right\}$, by [6, Corollary 7.5], we may suppose that $\mu \in\left(E^{\prime}\right)_{\mathcal{U}}$, and similarly, that $\lambda \in\left(F^{\prime}\right)_{\mathcal{U}}$, say $\mu=\left(\mu_{i}\right)$ and $\lambda=\left(\lambda_{i}\right)$. Pick representatives $x_{n}=\left(x_{n}^{(i)}\right)$ and $y_{n}=\left(y_{n}^{(i)}\right)$, so that by absolute convergence,

$$
\langle\mu \otimes \lambda, \tau\rangle=\lim _{i \rightarrow \mathcal{U}} \sum_{n=1}^{\infty}\left\langle\mu_{i}, x_{n}^{(i)}\right\rangle\left\langle\lambda_{i}, y_{n}^{(i)}\right\rangle=\left\langle\left(\mu_{i} \otimes \lambda_{i}\right), \psi_{0}(\tau)\right\rangle .
$$

Hence we must have that $\psi_{0}(\tau) \neq 0$.

Consequently, [3, Corollary 5.5] correctly shows that if $\mathcal{A}$ is a contractible Banach algebra with the approximation property, then $\mathcal{A}$ is ultra-amenable. However, a result of Selivanov, see [8, Theorem 4.1.5], shows that under these conditions, $\mathcal{A}$ is already the finite sum of full matrix algebras!

It is worth pointing out what can go wrong here (and hence the exact mistake in the proof of [3, Proposition 5.4]). If $\mathcal{A}$ is contractible, then we can find $\tau \in \mathcal{A} \widehat{\otimes} \mathcal{A}$ with $a \cdot \tau=\tau \cdot a$ and $\Delta(\tau) a=a$ for $a \in \mathcal{A}$. We can then treat $\tau$ as a member of $(\mathcal{A})_{\mathcal{U}} \widehat{\otimes}(\mathcal{A})_{\mathcal{U}}$, and we have that $a \cdot \psi_{0}(\tau)=\psi_{0}(\tau) \cdot a$ 
for $a \in(\mathcal{A})_{\mathcal{U}}$. As $\psi_{0}$ is an $(\mathcal{A})_{\mathcal{U}}$-module homomorphism, $\psi_{0}(a \cdot \tau-\tau \cdot a)=0$ for any $a \in(\mathcal{A})_{\mathcal{U}}$. However, if $\psi_{0}$ might fail to be injective, then this is not useful.

The following improves [3, Proposition 4.7], as a result of Grothendieck, see [9, Corollary 5.51], shows that a reflexive Banach space with the approximation property automatically has the metric approximation property.

Theorem A.3. If $(E)_{\mathcal{U}}$ has the bounded approximation property, then $\psi_{0}$ is bounded below.

Proof. Let $(E)_{\mathcal{U}}$ have the bounded approximation property with bound $M$, so by (the obvious generalisation of) [9, Theorem 4.14], the embedding $(E)_{\mathcal{U}} \widehat{\otimes}(F)_{\mathcal{U}} \rightarrow \mathcal{F}\left((E)_{\mathcal{U}},(F)_{\mathcal{U}}^{\prime}\right)$ is bounded below by $M^{-1}$. Here $\mathcal{F}\left((E)_{\mathcal{U}},(F)_{\mathcal{U}}^{\prime}\right)$ is the collection of finite-rank operators $(E)_{\mathcal{U}} \rightarrow(F)_{\mathcal{U}}^{\prime}$, given the operator norm.

Let $\tau \in(E)_{\mathcal{U}} \widehat{\otimes}(F)_{\mathcal{U}}$ have representative $\tau=\sum_{n=1}^{\infty} x_{n} \otimes y_{n}$. For $\epsilon>0$, we can find $T \in$ $\mathcal{F}\left((E)_{\mathcal{U}},(F)_{\mathcal{U}}^{\prime}\right)$ with $\|T\| \leq M+\epsilon$ and $|\langle T, \tau\rangle| \geq\|\tau\|$. Pick a representative

$$
T=\sum_{k=1}^{N} \mu_{k} \otimes \lambda_{k}
$$

for some $\left(\mu_{k}\right) \subseteq(E)_{\mathcal{U}}^{\prime}$ and $\left(\lambda_{k}\right) \subseteq(F)_{\mathcal{U}}^{\prime}$, so that

$$
\langle T, \tau\rangle=\sum_{n=1}^{\infty} \sum_{k=1}^{N}\left\langle\mu_{k}, x_{n}\right\rangle\left\langle\lambda_{k}, y_{n}\right\rangle .
$$

Let $G$ be the closed span of $\left\{x_{n}\right\}$, so by [6. Corollary 7.5], we can find a contraction $\phi: \operatorname{lin}\left\{\mu_{k}\right\} \rightarrow$ $\left(E^{\prime}\right) \mathcal{u}$ such that

$$
\left\langle\phi\left(\mu_{k}\right), x\right\rangle=\left\langle\mu_{k}, x\right\rangle \quad(1 \leq k \leq N, x \in G) .
$$

It's not hard to see that then

$$
T_{0}=\sum_{k=1}^{N} \phi\left(\mu_{k}\right) \otimes \lambda_{k}
$$

satisfies $\left\|T_{0}\right\| \leq M+\epsilon$ and $\left\langle T_{0}, \tau\right\rangle=\langle T, \tau\rangle$. In other words, we can assume that $\mu_{k} \in\left(E^{\prime}\right)_{\mathcal{U}}$ for each $k$; analogously, we may also assume that $\lambda_{k} \in\left(F^{\prime}\right)_{\mathcal{U}}$ for each $k$.

So, pick representatives $\mu_{k}=\left(\mu_{k}^{(i)}\right)$ and $\lambda_{k}=\left(\lambda_{k}^{(i)}\right)$, and for each $i$, let

$$
T_{i}=\sum_{k=1}^{N} \mu_{k}^{(i)} \otimes \lambda_{k}^{(i)} .
$$

As $\mathcal{U}$ is countably incomplete, we can find a sequence $\left(\epsilon_{i}\right)$ of strictly positive reals such that $\lim _{i \rightarrow \mathcal{U}} \epsilon_{i}=0$. For each $i$, pick $y_{i} \in E$ with $\left\|y_{i}\right\| \geq 1$ and $\left\|T_{i}\left(y_{i}\right)\right\| \geq\left\|T_{i}\right\|-\epsilon_{i}$. Let $y=\left(y_{i}\right)$ so $\|y\|=1$ and $T(y)=\left(T_{i}\left(y_{i}\right)\right)$ so that

$$
\lim _{i \rightarrow \mathcal{U}}\left\|T_{i}\right\|=\lim _{i \rightarrow \mathcal{U}}\left\|T_{i}\right\|-\epsilon_{i} \leq \lim _{i \rightarrow \mathcal{U}}\left\|T_{i}\left(y_{i}\right)\right\|=\|T(y)\| \leq(M+\epsilon) .
$$

Finally, a calculation shows that

$$
\langle T, \tau\rangle=\left\langle\left(T_{i}\right), \psi_{0}(\tau)\right\rangle,
$$

where $\left(T_{i}\right) \in\left(\mathcal{B}\left(E, F^{*}\right)\right)_{\mathcal{U}} \subseteq(E \widehat{\otimes} F)_{\mathcal{U}}^{\prime}$. We conclude that $\left\|\psi_{0}(\tau)\right\| \geq\|\tau\|(M+\epsilon)^{-1}$, so that $\psi_{0}$ is bounded below by $M^{-1}$. 
This shows that [3, Theorem 5.6] does give a correct characterisation of ultra-amenability for Banach algebras $\mathcal{A}$ whose ultrapowers have the bounded approximation property. This includes, for example, algebras of the form $L^{1}(G)$ for a locally compact group $G$.

Acknowledgments: I wish to thank: Seytek Tabaldyev who brought the problems in [4] (and hence also in [3]) to my attention; Volker Runde for pointing out the error in Theorem 5.7; and both Volker Runde and the authors of [7] for suggesting to look at polynomial identities as a way to fix Theorem 5.7.

\section{References}

[1] B. BlackAdAR, Operator algebras. Theory of $C^{*}$-algebras and von Neumann algebras. (Springer, Berlin, 2006).

[2] M. Brown and N. Ozawa, $C^{*}$-algebras and Finite-Dimensional Approximations (American Mathematical Society, Providence, RI, 2008).

[3] M. Daws, Amenability of ultrapowers of Banach algebras, Proc. Edinb. Math. Soc. (2) 52 (2009), 307-338.

[4] M. Daws And V. Runde, Can $\mathcal{B}\left(\ell^{p}\right)$ ever be amenable?, Studia Math. 188 (2005), 151-174.

[5] J. Dixmier, $C^{*}$-Algebras, (North-Holland Publishing Co., Amsterdam-New York-Oxford, 1977).

[6] S. HeinRich, Ultraproducts in Banach space theory, J. reine angew. Math. 313 (1980), 72-104.

[7] A. T.-M. Lau, R. J. Loy And G. A. Willis, Amenability of Banach and $C^{*}$-algebras on locally compact groups, Studia Math. 119 (1996), 161-178.

[8] V. Runde, Lectures on amenability (Springer, Berlin, 2002).

[9] R. RYAN, Introduction to tensor products of Banach spaces (Springer, London, 2002).

Matthew Daws, School of Mathematics, University of Leeds,

LEEDS LS2 9JT

United Kingdom

email: matt.daws@cantab.net 